The AstrophysicAL JournaL, 540:741-754, 2000 September 10

(c) 2000. The American Astronomical Society. All rights reserved. Printed in U.S.A.

\title{
HIGH-IONIZATION CLOUDS IN THE CIRCUMNUCLEAR REGION OF M31
}

\author{
C. Del Burgo, ${ }^{1,2,3}$ E. Mediavilla, ${ }^{1,2}$ AND S. Arribas ${ }^{1,4}$ \\ Received 1999 July 12; accepted 2000 April 13
}

\begin{abstract}
We present two-dimensional spectroscopy of the $125 \times 125 \mathrm{pc}^{2}$ circumnuclear region of M31, obtained using two optical fiber systems, INTEGRAL and 2D-FIS, installed at the $4.2 \mathrm{~m}$ William Herschel Telescope at the Roque de los Muchachos Observatory on the island of La Palma, Spain. We present continuum and emission-line maps, emission-line quotients, and stellar and ionized gas velocity fields obtained under subarcsecond seeing conditions. We have discovered $\mathrm{H} \beta$, [O III] $\lambda \lambda 4959,5007$, $\mathrm{H} \alpha$, and [N II] $\lambda \lambda 6548,6584$ emission in the inner $40 \times 40 \mathrm{pc}^{2}$ circumnuclear region. The intensity maps obtained from the emission lines show several isolated systems of ionized gas (clouds) a few parsecs in size. The kinematics of the clouds is decoupled from the stellar rotation, and strong radial motions are likely to be present. We have also discussed the ionization mechanisms present in the clouds. Although the emissionline quotients are compatible with ionization produced by post-asymptotic giant branch stars, the estimated ionization fluxes exceed what is expected according to the distribution of planetary nebulae in the bulge of M31.

Comparison of the line quotients and spectra found in M31 with those found in the extended narrowline region of the Seyfert galaxy NGC 4151 indicates that we could be in presence of ultralow-intensity nuclear activity. These results would be compatible with the existence of a "dead quasar" in the nucleus of M31 and underline the hybrid character of the LINER phenomenon.
\end{abstract}

Subject headings: galaxies: active - galaxies: individual (M31) — galaxies: kinematics and dynamics galaxies: nuclei — galaxies: Seyfert — instrumentation: spectrographs

\section{INTRODUCTION}

M31 is the nearest spiral $(\mathrm{Sb})$ galaxy $\left(1^{\prime \prime}=3.77 \mathrm{pc}\right.$, distance $\sim 778 \mathrm{kpc}$; Stanek \& Garnavich 1998). Owing to its proximity, M31 can be observed with a spatial resolution higher than that concerning any other galaxy beyond the Local Group. Thanks to this circumstance, two outstanding results concerning the inner central region have been obtained. First, from photometric observations of high spatial resolution (Light, Danielson, \& Schwarzschild 1974; Lauer et al. 1993; King, Stanford, \& Crane 1995) it is known that M31 has a double nucleus. Second, kinematic studies of the stellar component (Lallemand, Duschene, \& Walker 1960; Kormendy 1988; Dressler \& Richstone 1988; Bacon et al. 1994) have revealed the presence of a strong concentration of mass possibly associated with a supermassive black hole with over $10^{7} M_{\odot}$.

Complementary to the study of the stellar photometry and spectra, some spectroscopic features associated with the ionized gas have also been analyzed (Rubin \& Ford 1971; Ciardullo et al. 1988, hereafter C88), revealing the existence of a kiloparsec-scale central region of low surface emissionline gas of possible LINER characteristics (Heckman 1996, hereafter H96). LINERs offer the opportunity of studying the AGN phenomenon for ultralow luminosities, revealing new aspects of the inner environment of the central engine. This possibility is especially valuable for the nearby M31 in connection with the probable existence of a supermassive black hole (Kormendy \& Richstone 1995) in the peculiar

\footnotetext{
${ }^{1}$ Instituto de Astrofísica de Canarias, E-38200 La Laguna, Tenerife, Canary Islands, Spain.

${ }^{2}$ Departamento de Astrofísica de la Universidad de la Laguna, E-38206 La Laguna, Tenerife, Canary Islands, Spain.

3 cburgo@1l.iac.es.

${ }^{4}$ Consejo Superior de Investigaciones Científicas, Spain.
}

nucleus, and it lays great importance on the study of the ionized gas in the innermost central region. However, the weakness of the line emission has impeded until now the detection and study of the gaseous component close to the nucleus. Maybe as a consequence of this, there is no clear idea at present concerning what the source of photoionization in M31 could be (H96).

In this paper, we present two-dimensional spectroscopic data obtained with two optical fiber systems, 2D-FIS and INTEGRAL. In addition to a further refinement of previous observations (e.g., the stellar velocity field and continuum maps), they have allowed us to detect the weak and inhomogeneous ionized gas emission in the inner region of M31. In $\S 2$ we give details of the observations, the instrumentation used, and the data reduction procedures. The main results are presented in $\S 3$, and $\S 4$ is devoted to the discussion. Finally, in $\S 5$ we list our conclusions.

\section{OBSERVATIONS, INSTRUMENTATION, AND DATA REDUCTION}

The data analyzed in this paper were obtained during two different observing runs: 1994 September 14-15 and 1997 July 23, at the Roque de Los Muchachos Observatory (ORM) on the island of La Palma, Spain. During each one of these runs we used different instruments.

\subsection{D-FIS Observations}

In 1994 we used 2D-FIS, which links the $\mathrm{f} / 11$ Cassegrain focus of the $4.2 \mathrm{~m}$ William Herschel Telescope (WHT) with the ISIS double spectrograph. We will describe its main technical characteristics (for more details see García et al. 1994). The core of the system consists of a $2.5 \mathrm{~m}$ long bundle formed by 125 optical fibers, each $200 \mu \mathrm{m}$ in diameter $(0.9$ on the sky), arranged in two groups in the focal plane: (1) a central array $9 " .4 \times 12.2$ on the sky formed by 95 fibers and 
(2) 30 fibers forming a ring $38^{\prime \prime}$ in radius, which is intended for collecting background light (for small-sized objects). Figure 1 of Mediavilla et al. (1997) shows the distribution of the fibers in the telescope focal plane. The other ends of the fibers are arranged linearly along the spectrograph slit, allowing a set of 125 spectra to be recorded simultaneously for each exposure.

We used the blue and red arms of ISIS (Carter et al. 1993) simultaneously, equipped with 300 and 158 groove $\mathrm{mm}^{-1}$ gratings, respectively. In the blue (red), the mean dispersions were of $1.53(2.72) \AA$ pixel $^{-1}$ in the spectral range 4168-5754 (6121-9597) $\AA$. With this configuration four consecutive exposures of $1800 \mathrm{~s}$ were obtained from the central region of M31. Flat spectrum (image of a uniform white lamp passing through the bundle) and comparison lamp $(\mathrm{Cu}-\mathrm{Ar})$ images were also taken in order to perform the calibrations. The mean seeing was around 0"9.

\subsection{INTEGRAL Observations}

In 1997 we used the INTEGRAL fiber system, which links the f/11 Nasmyth focus of the $4.2 \mathrm{~m}$ WHT with the WYFFOS spectrograph. A detailed technical description of INTEGRAL is provided by Arribas et al. (1998); here we will only recall its main characteristics. INTEGRAL is
TABLE 1

PARAMETERS OF INTEGRAL BundLES

\begin{tabular}{cccc}
\hline \hline Bundle & $\begin{array}{c}D \\
(\operatorname{arcsec})\end{array}$ & $\begin{array}{c}\text { SC } \\
(\operatorname{arcsec} \times \operatorname{arcsec})\end{array}$ & $\mathrm{NF}(\mathrm{C}+\mathrm{R})$ \\
\hline SB1 $\ldots \ldots$ & 0.45 & $7.9 \times 6.7$ & $205(175+30)$ \\
SB2 $\ldots \ldots .$. & 0.90 & $16.4 \times 12.3$ & $219(189+30)$ \\
SB3 $\ldots \ldots$. & 2.70 & $34.4 \times 30.3$ & $135(115+20)$ \\
\hline
\end{tabular}

mounted on the GHRIL Nasmyth platform. It has three observing fiber bundles (called SB1, SB2, and SB3) of different sizes. Each bundle has the fibers arranged in two groups in the focal plane, one forming a rectangle and the other a ring of 90.2 in diameter. Table 1 presents the total number of fibers (rectangle plus ring) called $\mathrm{NF}(\mathrm{C}+\mathrm{R})$, the diameter $(D)$ of the fibers in arcseconds, and the spatial covering (SC) of the central rectangle for each one of the INTEGRAL bundles (see Fig. 1).

The WYFFOS spectrograph was equipped with the $\mathrm{R} 1200 \mathrm{~B}$ grating (1200 grooves $\mathrm{mm}^{-1}$ ), which allowed us to obtain a mean dispersion of $1.45 \AA$ pixel $^{-1}$ in the spectral range $4330-5830 \AA$. With this configuration, two exposures of $1200 \mathrm{~s}$ using SB1 and SB2 and one exposure of $1000 \mathrm{~s}$
SB1
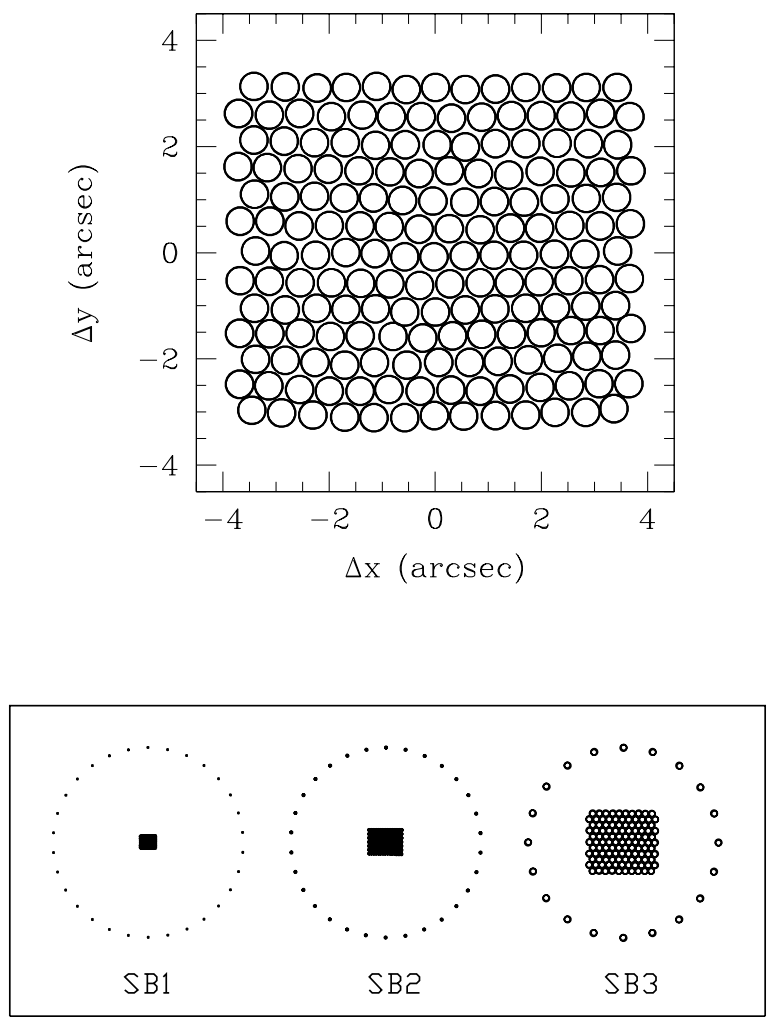

SB2

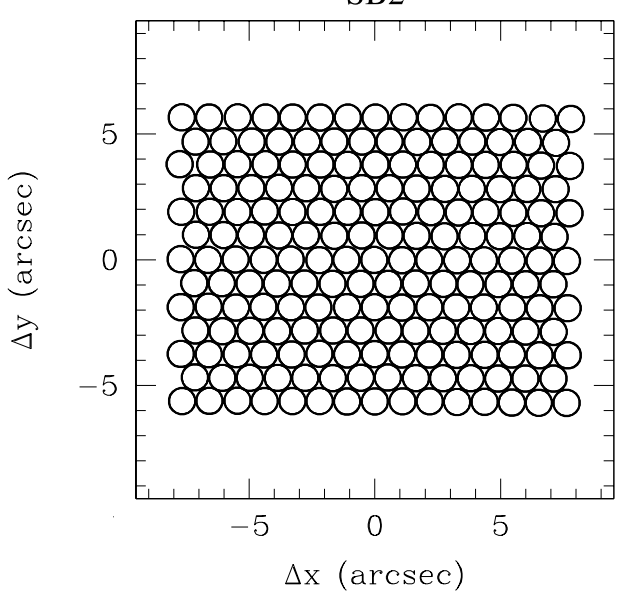

SB3

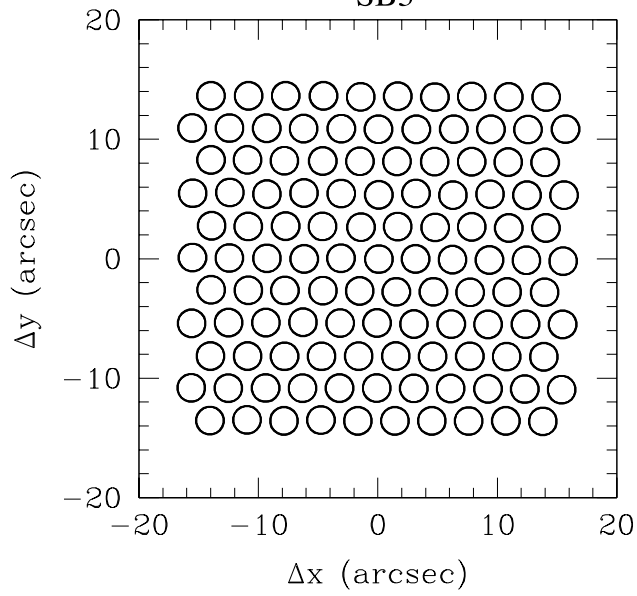

FIG. 1.-Spatial distribution of the fiber faces of the central rectangle at the focal plane end for the INTEGRAL bundles SB1 (top left), SB2 (top right), and SB3 (bottom right), and a schematic overview of these including the fibers of the external ring. 
using SB3 were obtained from the central region of M31. In addition, two exposures of a velocity template star (HR 8665) were obtained using SB1 and SB2. At the end of the observing night several twilight images, which contain the solar spectrum (scattered from the twilight sky), were obtained using the SB3 bundle. Images of flat spectrum and comparison lamps $(\mathrm{Cu}-\mathrm{Ar})$ were also obtained in order to perform the corresponding calibrations. The mean seeing was around 0.7 .

All bundles (SB1, SB2, SB3, and 2D-FIS) were approximately centered on the nucleus of M31.

\subsection{Data Reduction}

The data reduction procedure includes the following steps: bias subtraction, aperture definition and trace, straylight subtraction, the extraction of the spectra, wavelength calibration, throughput correction, and cosmic-ray rejection.

To obtain the two-dimensional maps presented in this paper we regularly interpolated the selected parameter (e.g., emission-line intensity, mean radial velocity), with the help of some NAG routines from the grid of values defined by the fiber bundle.

\section{RESULTS}

\subsection{Emission-Line Features}

Figure 2 shows the reduced spectra in the range 4940 $5027 \AA$ with the $[\mathrm{O}$ III] $\lambda \lambda 4959,5007$ emission lines for the SB1 (Fig. 2a), SB2 (Fig. 2b), and SB3 (Fig. 2c) data. In Figure 3, the 2D-FIS spectra in the ranges of the [O III] $\lambda \lambda 4959,5007$ (Fig. $3 a$ ) and $\mathrm{H} \alpha+[\mathrm{N} \mathrm{II}] \lambda \lambda 6548,6584$ (Fig. $3 b$ ) emission lines are shown.

An outstanding feature of these data is the presence of weak emission lines in some spectra. In the figure corresponding to the SB2 bundle of INTEGRAL (Fig. 2b), for instance, we identify relatively strong [O III] emission lines in two regions, one corresponding to spectra 95, 103, and 104 , and the other to spectra 45,62 , and 63 . Both regions can also be recognized in Figure $3 a$ (data obtained using 2D-FIS), which shares a common area with Figure $2 b$. In the $\mathrm{H} \alpha+[\mathrm{N} \mathrm{II}] \lambda \lambda 6548,6584$ spectral region (Fig. $3 b$ ) the strong $\mathrm{H} \alpha$ absorption complicates the identification of emission-line features; these can nevertheless be clearly identified in several spectra $(10,13,14,19,20,21,29,30$, and 42 at the top, 48 in the middle, and 102 at the bottom).

The lack of appreciable emission in the nucleus and the inhomogeneous distribution explain why this emission has hitherto passed unnoticed in long-slit studies. It is also important to point out the unsuitability of narrowband imaging to isolate the line emission in galaxies (like M31) where the emission is very weak with respect to the stellar continuum.

\subsection{The Stellar Component: Morphology and Kinematics}

In Figure 4 we present a map of the stellar continuum obtained by integrating the spectra corresponding to the INTEGRAL bundle with highest spatial sampling (SB1) in the $5600-5700 \AA$ wavelength range. Thanks to the good seeing and instrumental performance, the presence of the double nucleus is easily appreciated. Adopting the usual convention, we name the brightest point in this map P1. P2 is the secondary nucleus, which we have assumed to coincide with the center of the outermost isophotes (we have selected the isophote with a semimajor axis of 2".5). It has been noted by previous authors that the intensity quotient between $\mathrm{P} 1$ and $\mathrm{P} 2$ depends on wavelength, P2 being brighter at $175 \mathrm{~nm}$ (King et al. 1995). Thus, the location of the intensity peak can change with the wavelength. Profiting from the large and continuous spectral range observed with 2D-FIS, we have built up several continuum maps at different wavelengths (see Fig. 5). In this case, the error in the intensity peak location is lower than 0"3. From this collection of maps we confirm that the quotient P2/P1 increases toward the blue, with a much larger contribution of P2 toward wavelengths lower than $4100 \AA$. Lauer et al. (1998) have reported that the crossover must be between 3000 and $5550 \AA$, but according to our data it takes place near $5650 \AA$. From the $9100-9200 \AA$ continuum map we estimate a separation between the two nuclei of 0".45 [0".49 from the F555W image taken with the Hubble Space Telescope (HST) Wide Field Planetary Camera; Lauer et al. 1993].

Figure 6 shows the stellar velocity fields obtained with the SB1 (Fig. 6a) and SB2 (Fig. 6b) bundles of INTEGRAL derived by cross-correlating the spectra in the range 5100 $5400 \AA$. Although the central region of M31 is photometrically dominated by the bulge, according to the stellar velocity map the stars seem to be forming a disk rotating very rapidly around $\mathrm{P} 2$. Out of the central region $\left(r>2^{\prime \prime}\right)$ the stellar radial velocity falls to the systemic velocity of M31 (-300 $\left.\mathrm{km} \mathrm{s}^{-1}\right)$. The peak-to-peak amplitude of the velocity field in the map corresponding to the highest spatial resolution (SB1; Fig. $6 a$ ) is $284 \mathrm{~km} \mathrm{~s}^{-1}$, and the separation between the minimum and the maximum is 1".58. Bacon et al. (1994) found $240 \mathrm{~km} \mathrm{~s}^{-1}$ for the amplitude and 1 1"89 for the separation, and Statler et al. (1999), from $\mathrm{f} / 48$ long-slit data of the HST Faint Object Camera along the P1-P2 line (P.A. $=42^{\circ}$ ) with a slit width of 0.063, have reported an amplitude of around $500 \mathrm{~km} \mathrm{~s}^{-1}$ and a separation of 0.6. The differences among these authors could be due mainly to different spatial resolutions and seeing conditions.

From SB1 data we have also derived the map of velocity dispersions. We found a maximum (S) of $258 \mathrm{~km} \mathrm{~s}^{-1}$ offset 0".45 southwest from P2 (see Fig. 7). Bacon et al. (1994) found 0.73 for this distance. However, in the better spatial resolution work of Statler et al. (1999) it is found that the velocity dispersion peak $\left(440 \pm 70 \mathrm{~km} \mathrm{~s}^{-1}\right)$ almost coincides with P2 (at a distance of $0{ }^{\prime \prime} 06 \pm 0$ 0 03 ). Despite the better spatial resolution in the case of Statler et al. (1999), it is not possible to explain such a difference at the location of $\mathrm{S}$ in terms of spatial resolution and seeing. It is likely that the slit position of Statler et al. (1999) would not pass through the maximum detected by us (see also Bacon et al. 1994). In fact, P1, P2, and S are not exactly along the same axis. Maybe there are two local maxima at the center of M31, one of these at the center and the other one shifted around 0.5 toward the southwest. It should be very interesting to perform new observations with $H S T$ to resolve this puzzle.

We will make use of the information on the stellar kinematics in the following sections to compare with that of the ionized gas.

\subsection{Spectra Corrected for Absorption}

In order to obtain the emission-line spectra free of absorption lines, we subtract a weighted mean absorption 


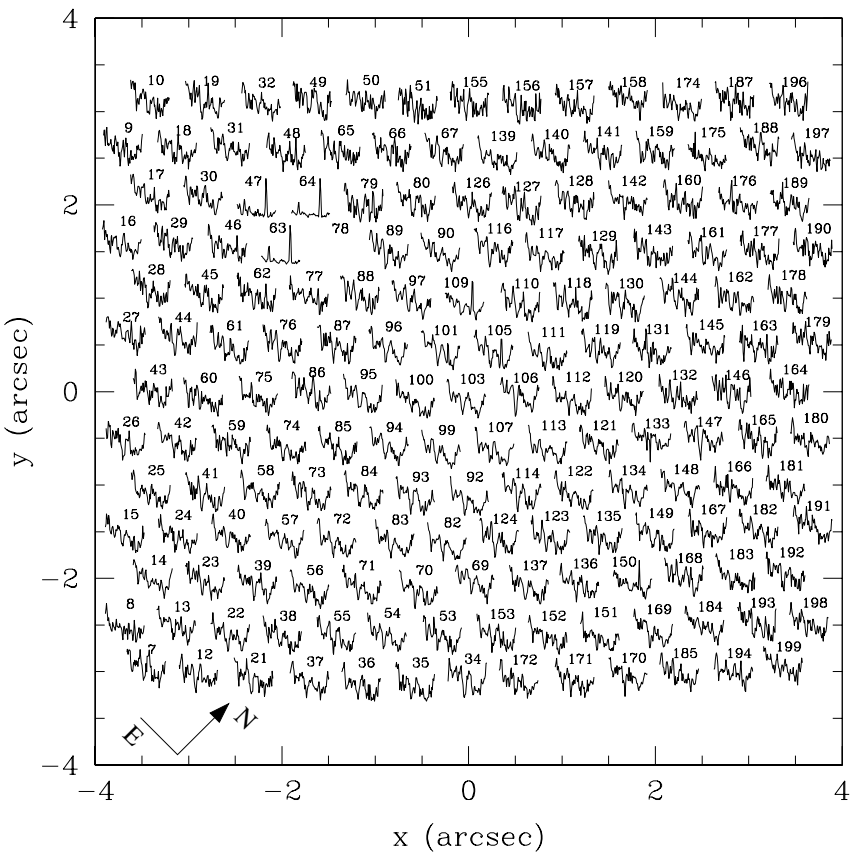

FIG. $2 a$

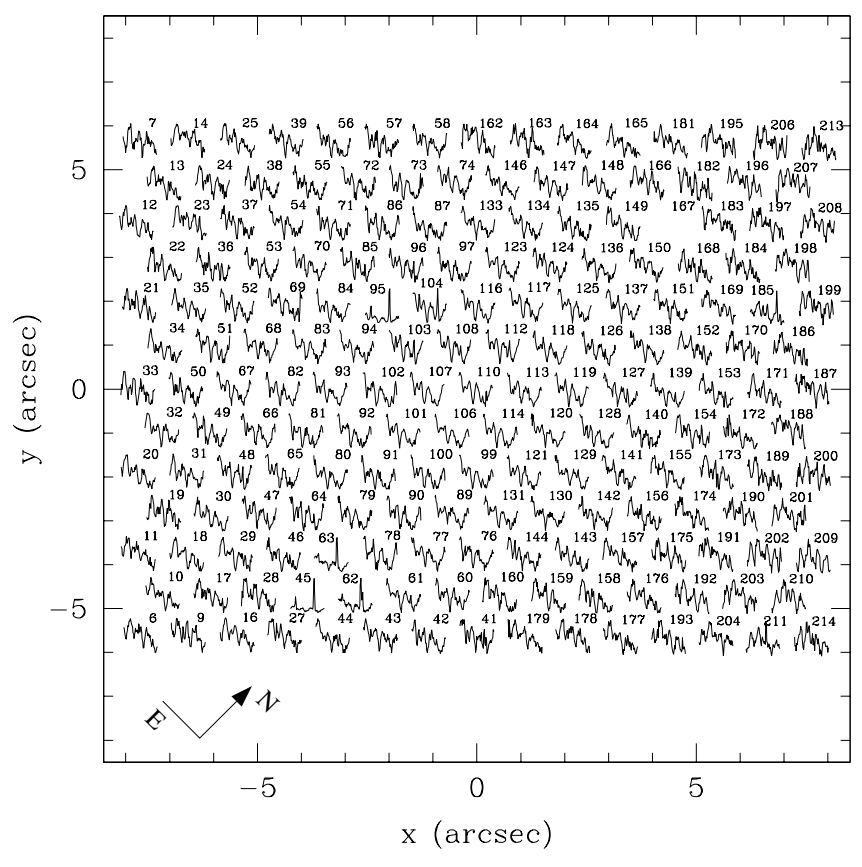

FIG. $2 b$

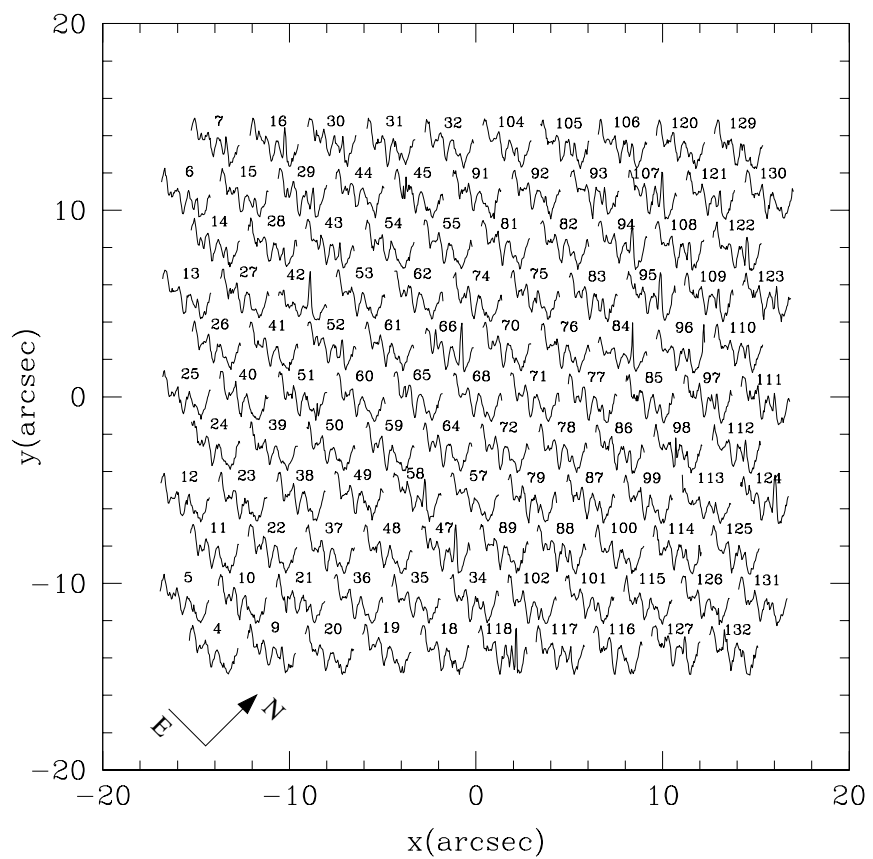

FIG. $2 c$

FIG. 2.-M31 spectra obtained using the SB1 $(a), \mathrm{SB} 2(b)$, and SB3 $(c)$ bundles. Note the $[\mathrm{O} \mathrm{III}] \lambda \lambda 4959,5007$ emission lines. Orientation: north/east is indicated by an arrow with/without head.

spectrum according to the following procedure (for each one of the bundles): (1) we correct the relative shifts produced by the stellar velocity field of M31 for each of the spectra, (2) we obtain a mean absorption spectrum by summing all the spectra without emission lines, and (3) we weight this mean spectrum (template) by the spectrum/template flux ratio on both sides of the emission lines and subtract it from each spectrum. One potential drawback of this procedure could be that the mean absorption spectra (template) subtracted were not representative at the location of the clouds. However, by generating templates with different sets of spectra we have checked that the absorption spectra are indistinguishable over the observed region.

After following this procedure we inspected the corrected spectra and found line emission in some regions. To illustrate the method and the results, in Figure 8 we present the spectra taken with 2D-FIS in the 4840-5025 $\AA$ and 6500 $6640 \AA$ A spectral ranges, before and after subtraction of the template absorption spectrum.

The emission lines of the absorption-free spectra were fitted by Gaussians. First, we select the emission lines $\mathrm{H} \beta$ and $[\mathrm{O} \mathrm{III}] \lambda \lambda 4959,5007$ in the blue region and fit them 


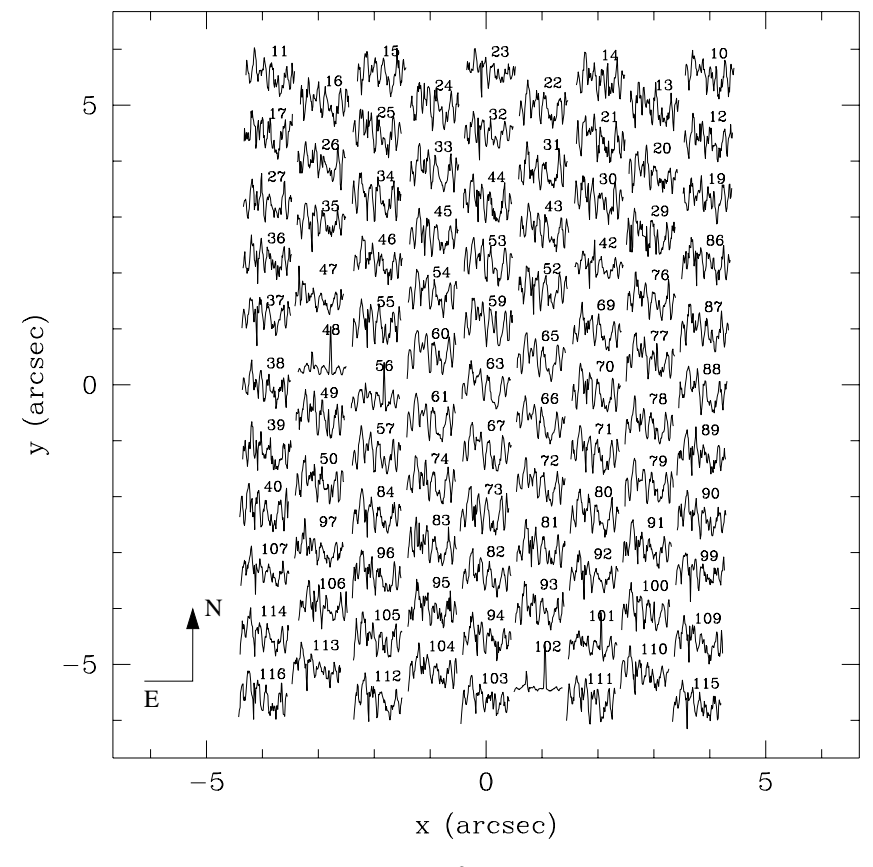

FIG. $3 a$

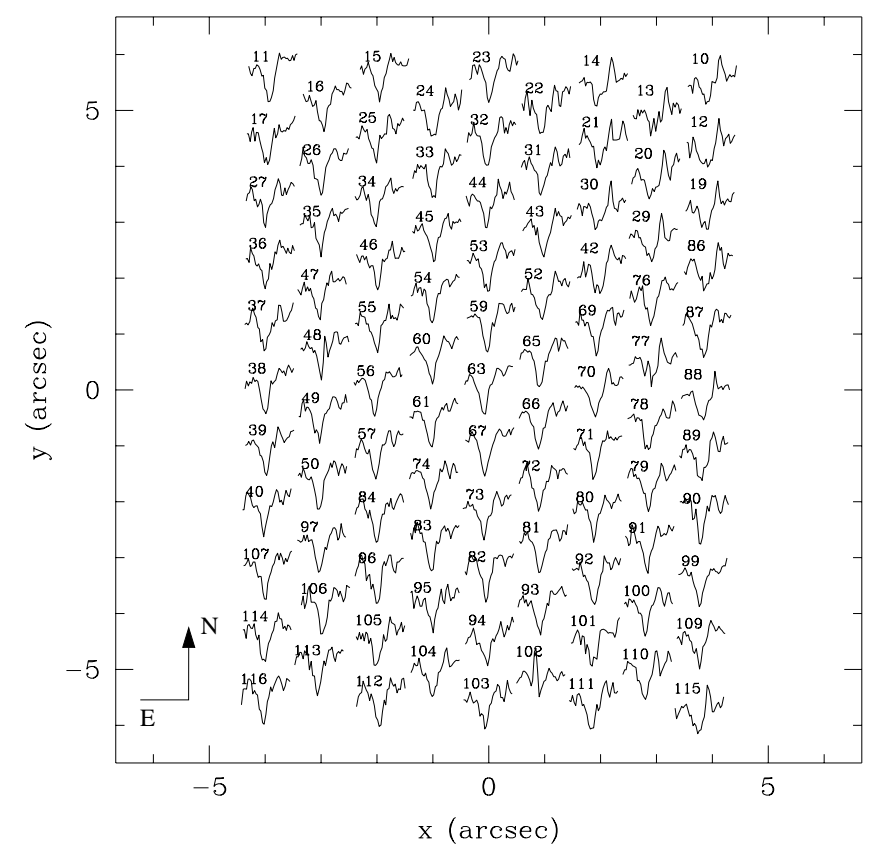

FIG. $3 b$

FIG. 3.-(a) M31 spectra obtained using 2D-FIS in the spectral range close to [O III] $\lambda \lambda 4959,5007$ emission lines; (b) M31 spectra with $\mathrm{H} \alpha$ and [N II] $\lambda \lambda 6548,6584$ emission lines. These spectra correspond to the image obtained with the lowest air mass. Orientation: north/east is indicated by an arrow with/without head.

with the constraints $I_{\left[\mathrm{O}_{\mathrm{II}}\right] \lambda 5007} / I_{\left[\mathrm{O}_{\mathrm{II}}\right] \lambda 4959}=3$ and $\mathrm{FWHM}_{\left[\mathrm{O}_{\mathrm{III}}\right] 25007}=\mathrm{FWHM}_{[\mathrm{O} \text { m] }] \lambda 4959}$. In the same way, we fit the $\mathrm{H} \alpha$ and $[\mathrm{N}$ II] $\lambda 6548,6584$ emission lines with the constraints $I_{[\mathrm{N} \mathrm{I}] 26584} / I_{[\mathrm{N} \mathrm{m}] 26548}=3$ and $\mathrm{FWHM}_{[\mathrm{N} \mathrm{II]} \lambda 6584}=\mathrm{FWHM}_{[\mathrm{N} \mathrm{I}] \lambda 6548}$. From these Gaussian fits, we have obtained the mean velocities, velocity dispersions (after correction of the instrumental width), and emission-line ratios corresponding to different ionized gas regions, which will be identified in the next section. These quotients have been corrected from extinction and the whole system throughput depending on the wavelength. There is no evidence for dust extinction greater than 0.2 mag in the central 15" (Bacon et al. 1994). The extinction in $V$ is $A_{V}=0.24$ according to Burstein \& Heiles (1984). From this value and the extinction ratios of Mathis (1990) we have determined that $A_{\mathrm{H} \beta}=0.28$ and $A_{[\mathrm{O} \mathrm{ml} \lambda 5007}=0.27$. Thus, $\mathrm{H} \beta$ is $4 \%$ more reddened than [O III] $\lambda 5007$. Regarding the system response, it is $10 \%$ lower at $\mathrm{H} \beta$ than at [O III] $\lambda 5007$ for INTEGRAL and 2D-FIS. No significant difference is given between [N II] $\lambda 6584$ and $\mathrm{H} \alpha$. We discuss a larger reddening at the locations of the clouds in $\S 4$. Table 2 presents these values for the 2D-FIS and INTEGRAL data.

\subsection{Identification of Ionized Gas Systems}

In Figure 9 we present the intensity maps of the $\mathrm{H} \beta$, [O III] $\lambda 5007$, [N II] $\lambda 6584$, and $\mathrm{H} \alpha$ emission lines corresponding to the observations made with 2D-FIS. Figures 10,11 , and 12 show the intensity map of the [O III] $\lambda 5007$ and $\mathrm{H} \beta$ emission lines corresponding to the three (SB1, SB2, and SB3) bundles of INTEGRAL.

If we look at Figure 10 (corresponding to SB1) we can identify three isolated regions (ionized gas systems or clouds: A, B, and C) of relatively small size and roughly aligned along the east-west axis. These clouds also appear in the intensity maps of Figure 11, where two more clouds,
$\mathrm{D}$ and $\mathrm{E}$, are present in addition. In the inner region of Figure 12 we can recognize, blurred by the low spatial resolution, clouds A, B, D, and E. Other features of spread or compact emission (regions $\mathrm{G}$ and $\mathrm{F}$ ) also appear in the outer region of this figure.

From the data of Table 2 we have inferred the mean parameters for the clouds A, B, C, D, E, F, and G (see Table 3). To estimate the cloud sizes we have used the expression

$$
\mathrm{FWHM}=\left(\mathrm{FWHM}_{0}^{2}-\mathrm{FWHM}_{s}^{2}\right)^{1 / 2},
$$

where $\mathrm{FWHM}_{0}$ corresponds to the measured size of the cloud and $\mathrm{FWHM}_{s}$ is the seeing that we have computed by comparing the photometric models of Bacon et al. (1994) with our continuum in $5600 \AA$. The resulting sizes for regions $\mathrm{A}, \mathrm{D}, \mathrm{F}, \mathrm{G}$, and $\mathrm{E}$ are included in Table 3. Clouds $\mathrm{B}$ and $\mathrm{C}$ are unresolved.

\subsection{Emission-Line Ratios}

From the Gaussian fits to the emission lines ( $\S 3.3$ ) we have inferred the mean [O III] $\lambda 5007 / \mathrm{H} \beta$ and [N II] $\lambda 6584 /$ $\mathrm{H} \alpha$ ratios for the clouds A, B, C, D, and E (see Table 3). As we compared the data obtained with two different instruments in two different observations, the uncertainties in the [O III] $\lambda 5007 / \mathrm{H} \beta$ quotients are quite reliable. This quotient indicates that the ionization in clouds $\mathrm{A}, \mathrm{B}$, and $\mathrm{D}$ is higher than expected in LINERs. The [N II] $\lambda 6584 / \mathrm{H} \alpha$ was obtained exclusively from the data obtained with 2D-FIS, and we do not have a good estimate of the uncertainty except in the case of region $\mathrm{E}$, which is a strong emitter in [N II] $\lambda 6584+\mathrm{H} \alpha$, and we obtained the mean from nine values.

As can be seen in Table 2, cloud D probably corresponds to at least two different kinematic subsystems at velocities of approximately -270 and $-527 \mathrm{~km} \mathrm{~s}^{-1}$, respectively. 


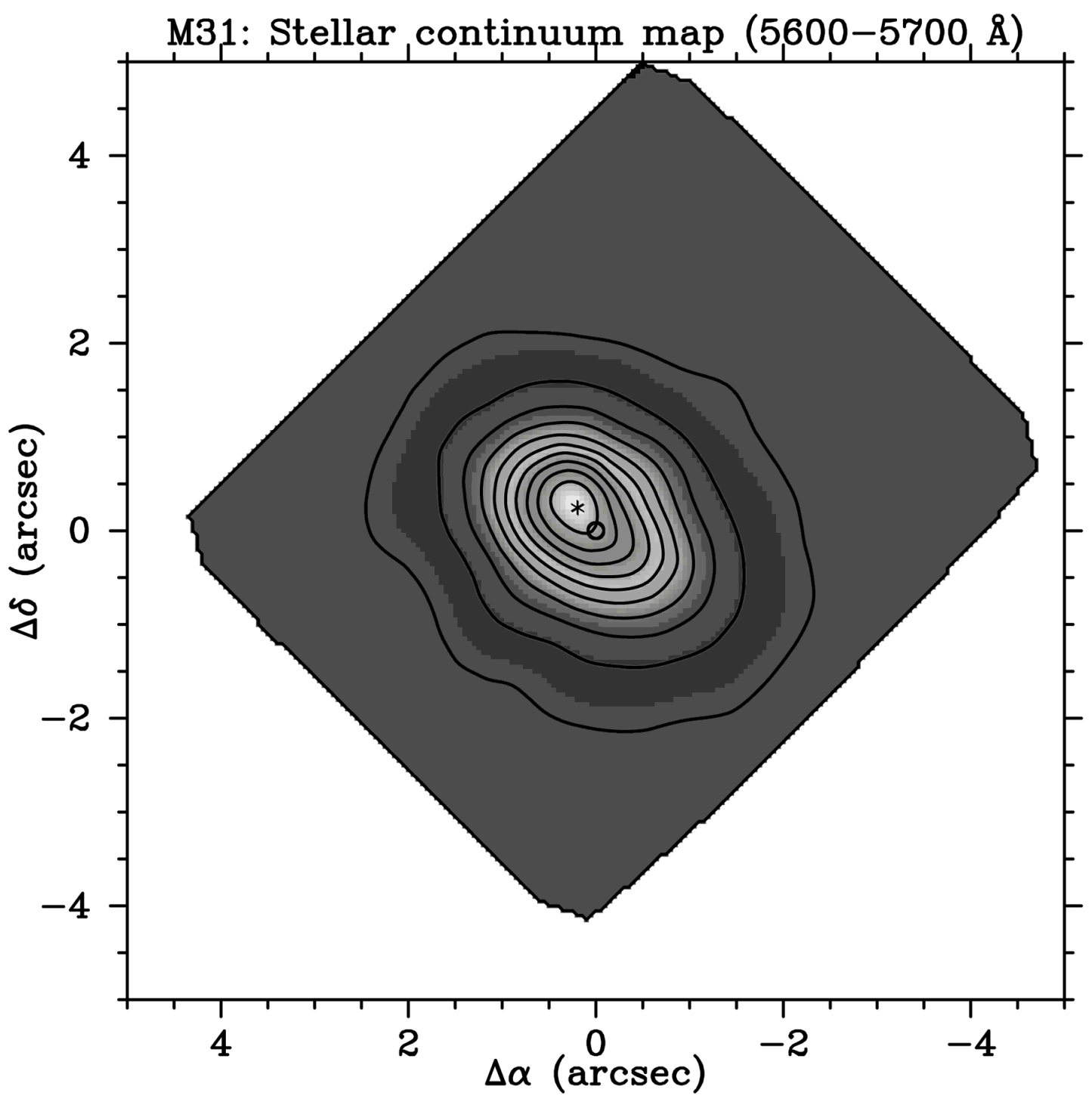

FIG. 4. - Continuum map in the range 5600-5700 ̊ obtained using the SB1 bundle. Note the offset between the secondary peak, P2 (circle), and the brightest peak, P1 (asterisk).

This result is clearly manifest in the spectra corresponding to fiber 62 of the SB2 bundle of INTEGRAL, where the emission lines appear split into two components. (A third determination of velocity, approximately $-362 \mathrm{~km} \mathrm{~s}^{-1}$, corresponding to fiber 34 of SB3 could indicate the existence of a gradient but could also be due to the lower signalto-noise ratio of this spectrum.) In any case, from our data we have no reason to suppose that different ionization con-
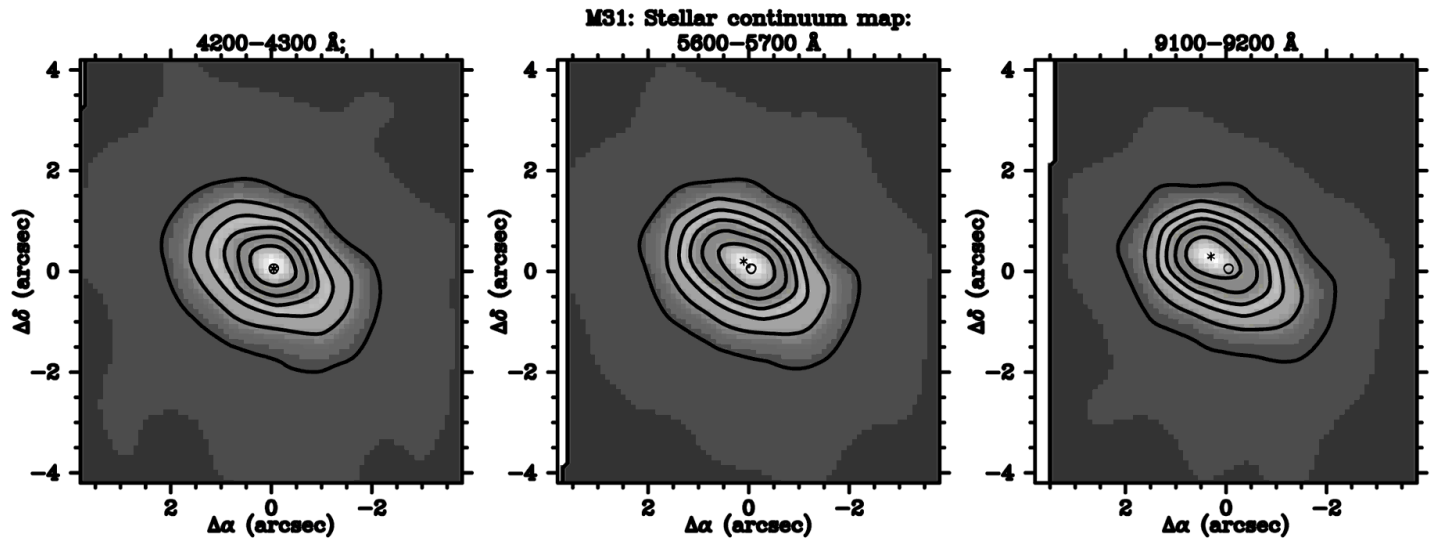

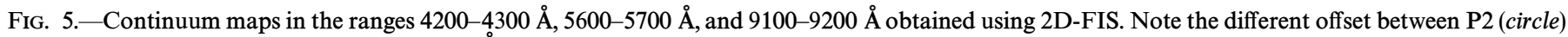
and the maxima (asterisk) at 4250, 5650, and $9150 \AA$. 


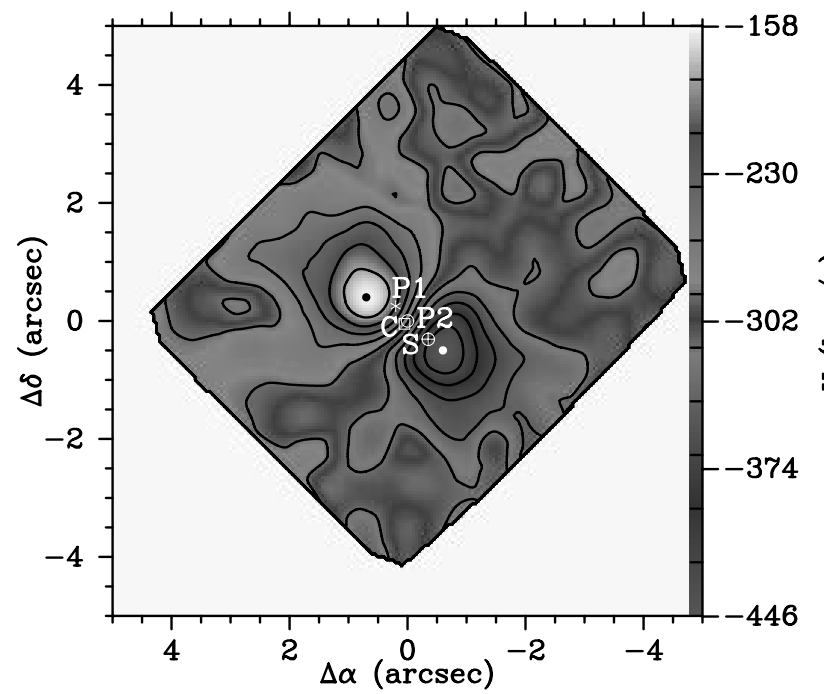

FIG. $6 a$

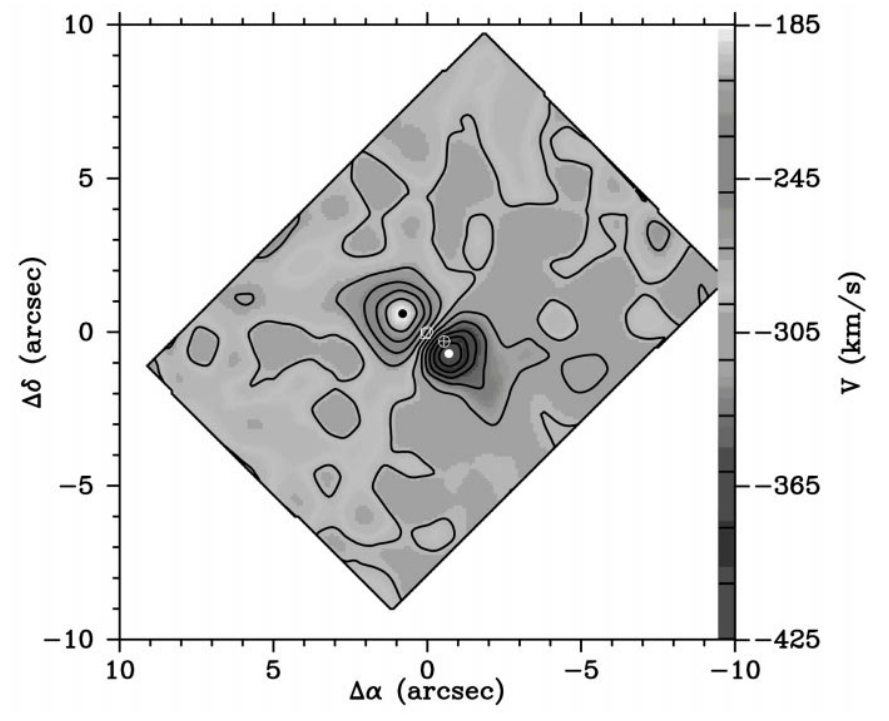

FIG. $6 b$

FIG. 6. - (a) Stellar velocity field obtained from a cross-correlation of the SB1 spectra between 5100 and $5400 \AA$ A; (b) stellar velocity field obtained from a cross-correlation of the SB2 spectra in the same range. The points marked are the stellar kinematic center C (square), the stellar velocity dispersion maximum $\mathrm{S}$ (circle with a plus inside), P2 (circle), the location of P1 at $5650 \AA$ (asterisk), and the maximum and minimum of stellar velocity (black and white point, respectively). [See the electronic edition of the Journal for a color version of this figure.]

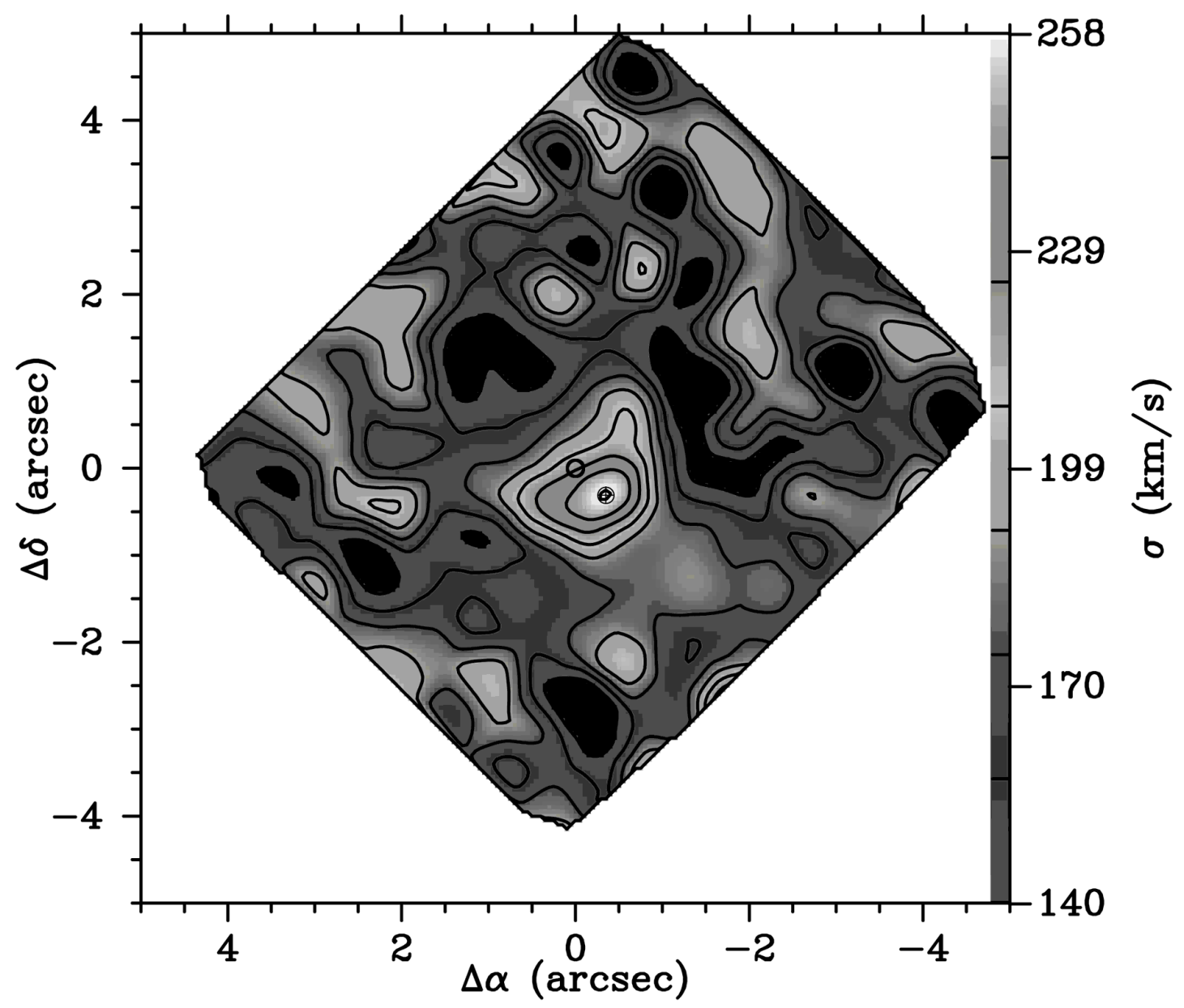

FIG. 7.- Stellar dispersion velocity field obtained from a cross-correlation of the SB1 spectra between 5100 and $5400 \AA \AA$. The points stand for P2 (circle) and $\mathrm{S}$ (circle with a plus inside). 


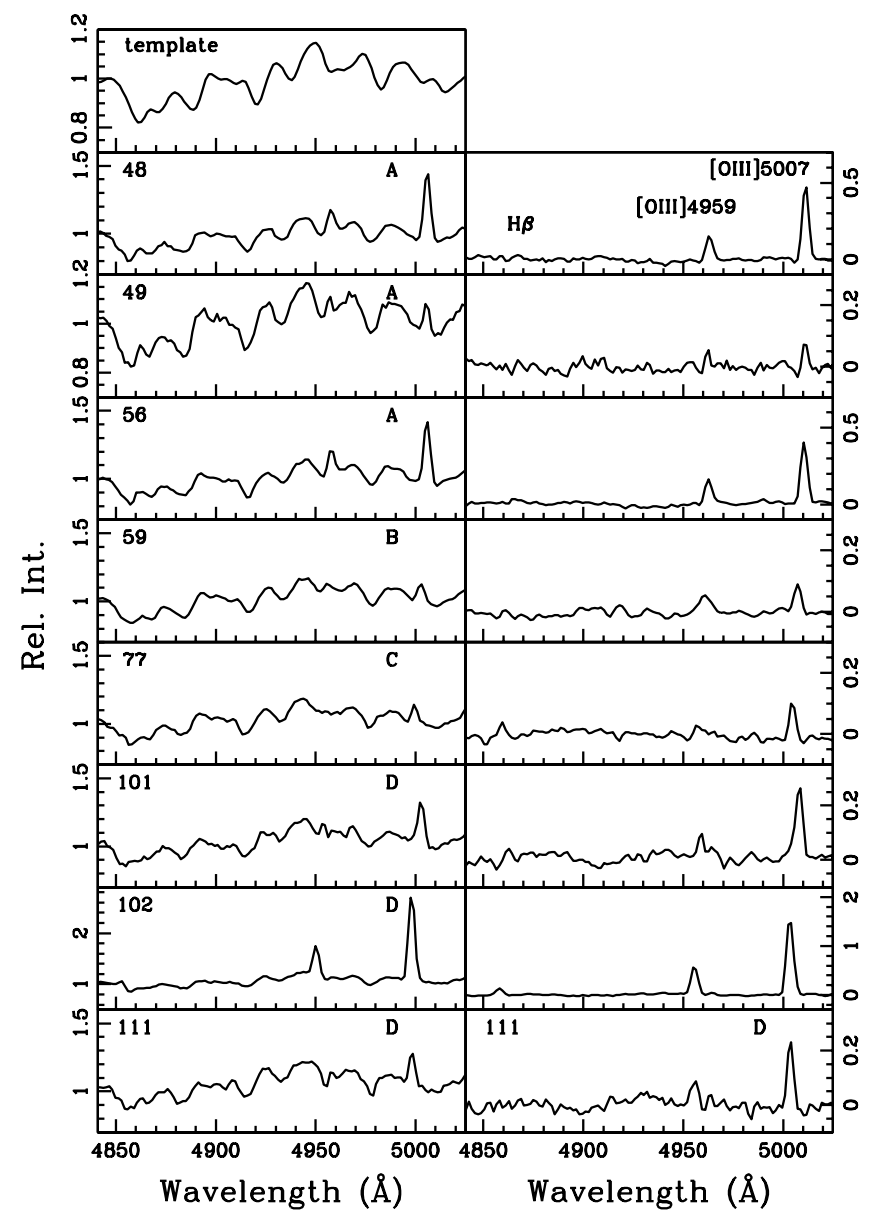

FIG. $8 a$

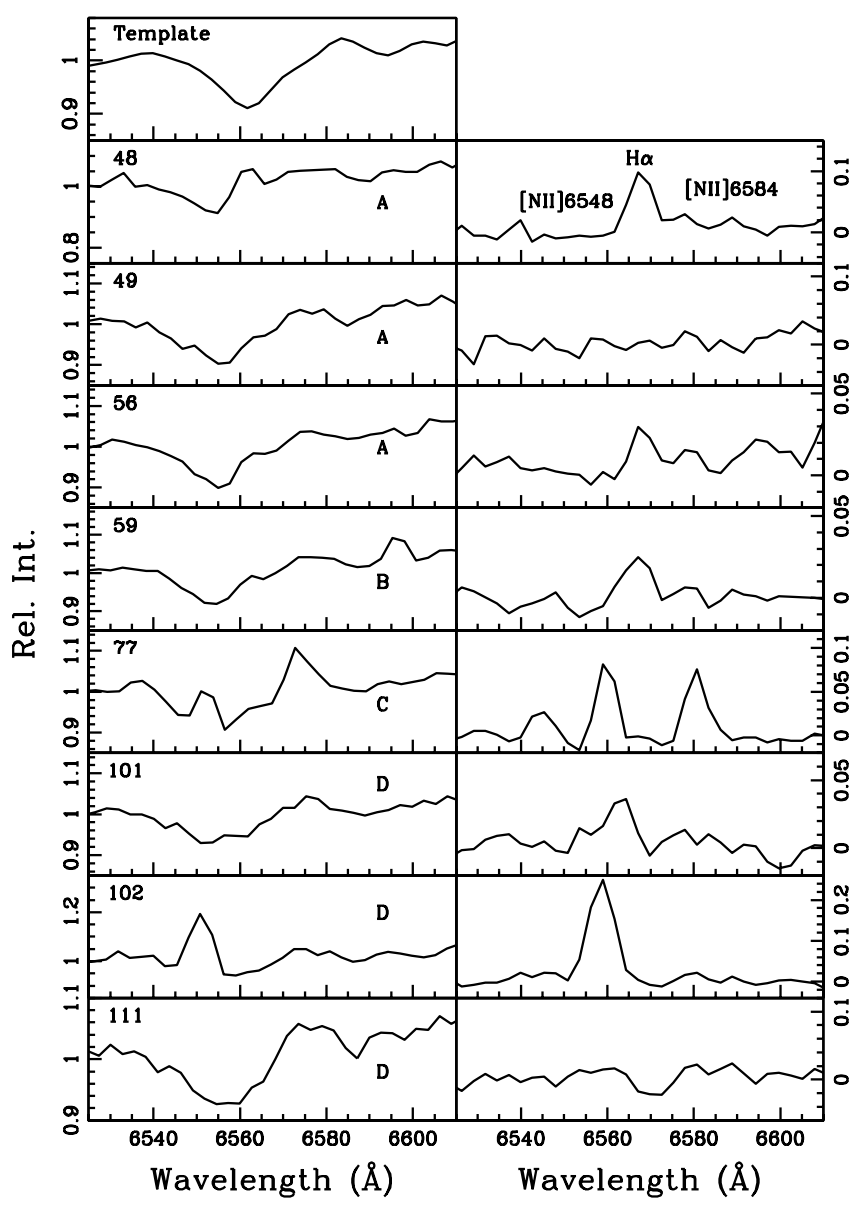

FIG. $8 b$

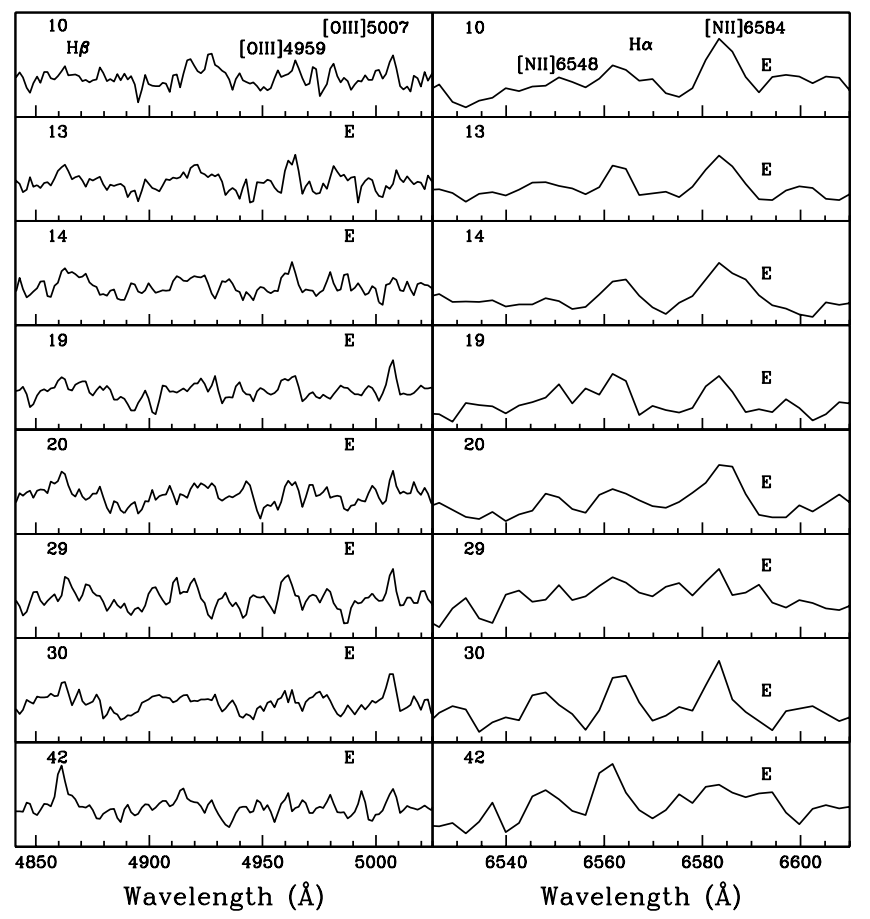

FIG. $8 c$

FIG. 8.-(a) Spectra in the range of $\mathrm{H} \beta$ and [O III] $\lambda \lambda 4959,5007$ concerning the identified ionized gas clouds and regions. At the top is shown the absorption template. Right: spectra after subtraction. (b) Same as (a), but in the range with $\mathrm{H} \alpha$ and [N II] $\lambda \lambda 6548$, 6584. (c) Spectra with $\mathrm{H} \beta$ and [O III] $\lambda \lambda 4959,5007$ for ionized gas region E. Right: spectra with $\mathrm{H} \alpha$ and $[\mathrm{N} \mathrm{II}] \lambda 6548,6584$ for this region. (2D-FIS data corresponding to the combined image.) 
TABLE 2

Characteristic Parameters of the IONIZed GaS

\begin{tabular}{|c|c|c|c|c|c|c|c|c|c|c|c|}
\hline $\begin{array}{c}\text { Region } \\
\text { (fiber) }\end{array}$ & $\begin{array}{c}\Delta \alpha^{\mathrm{a}} \\
(\operatorname{arcsec})\end{array}$ & $\begin{array}{c}\Delta \delta^{\mathrm{a}} \\
(\operatorname{arcsec})\end{array}$ & Bundle & $\begin{array}{c}V_{\text {gas }} \\
\left(\mathrm{km} \mathrm{s}^{-1}\right)\end{array}$ & $\begin{array}{c}V_{\text {star }} \\
\left(\mathrm{km} \mathrm{s}^{-1}\right)\end{array}$ & $\begin{array}{c}\sigma(\mathrm{H} \beta) \\
\left(\mathrm{km} \mathrm{s}^{-1}\right)\end{array}$ & $\begin{array}{c}\sigma(\mathrm{H} \alpha) \\
\left(\mathrm{km} \mathrm{s}^{-1}\right)\end{array}$ & $\begin{array}{l}\sigma([\mathrm{O} \mathrm{III}]) \\
\left(\mathrm{km} \mathrm{s}^{-1}\right)\end{array}$ & $\begin{array}{l}\sigma([\mathrm{N} \mathrm{II}]) \\
\left(\mathrm{km} \mathrm{s}^{-1}\right)\end{array}$ & {$[\mathrm{O} \mathrm{III}] \lambda 5007 / \mathrm{H} \beta$} & {$[\mathrm{N} \mathrm{II]}] \lambda 6584 / \mathrm{H} \alpha$} \\
\hline $\mathrm{A}(48) \ldots \ldots$. & 2.7 & 0.6 & 2D-FIS ${ }^{b}$ & -51 & -315 & $93 \pm 31$ & $63 \pm 9$ & $48 \pm 1$ & $\ldots$ & $12 \pm 4$ & $0.14 \pm 0.10$ \\
\hline$A(56) \ldots \ldots$. & 1.7 & 0.0 & 2D-FIS & -57 & -278 & $\ldots$ & $\ldots$ & $65 \pm 2$ & $\ldots$ & $9 \pm 2$ & $\ldots$ \\
\hline$A(47) \ldots \ldots$. & 2.8 & 0.2 & $\mathrm{SB} 1^{\mathrm{c}}$ & -59 & -319 & $\ldots$ & $\ldots$ & $66 \pm 3$ & $\ldots$ & $\ldots$ & $\ldots$ \\
\hline$A(63) \ldots \ldots$ & 2.3 & 0.0 & SB1 & -56 & -294 & $59 \pm 20$ & $\ldots$ & $59 \pm 1$ & $\ldots$ & $13 \pm 5$ & $\ldots$ \\
\hline A (64) ........ & 2.4 & 0.7 & SB1 & -60 & -278 & $\ldots$ & $\ldots$ & $58 \pm 1$ & $\ldots$ & $\ldots$ & $\ldots$ \\
\hline$A(95) \ldots \ldots$. & 2.6 & 0.1 & $\mathrm{SB}^{\mathrm{d}}$ & -67 & -283 & $\ldots$ & $\ldots$ & $60 \pm 1$ & $\ldots$ & $\ldots$ & $\ldots$ \\
\hline$A(103) \ldots \ldots$ & 1.5 & -0.1 & SB2 & -54 & -239 & $\ldots$ & $\ldots$ & $64 \pm 11$ & $\ldots$ & $\ldots$ & $\ldots$ \\
\hline A $(104) \ldots \ldots$ & 1.8 & 0.9 & SB2 & -46 & -240 & $\ldots$ & $\ldots$ & $45 \pm 3$ & $\ldots$ & $10 \pm 5$ & $\ldots$ \\
\hline B (59) ........ & -0.3 & 1.1 & 2D-FIS & -258 & -288 & $\ldots$ & $70 \pm 25$ & $73 \pm 7$ & $\ldots$ & $12 \pm 8$ & $0.15 \pm 0.11$ \\
\hline B (109) ...... & 0.4 & 1.0 & SB1 & -273 & -207 & $\ldots$ & $\ldots$ & $62 \pm 6$ & $\ldots$ & $10 \pm 6$ & $\ldots$ \\
\hline B $(112) \ldots \ldots$ & 0.0 & 1.4 & SB2 & -259 & -265 & $\ldots$ & $\ldots$ & $\ldots$ & $\ldots$ & $\ldots$ & $\ldots$ \\
\hline $\mathrm{C}(77) \ldots \ldots$ & -3.2 & 0.5 & 2D-FIS & -450 & -324 & $\ldots$ & $\ldots$ & $\ldots$ & $\ldots$ & $2.6 \pm 0.9$ & $1.0 \pm 0.2$ \\
\hline$C(150) \ldots \ldots$ & -2.9 & 0.2 & SB1 & -470 & -296 & $\ldots$ & $\ldots$ & $58 \pm 6$ & $\ldots$ & $2.0 \pm 0.7$ & $\ldots$ \\
\hline$C(129) \ldots \ldots$ & -3.1 & 0.5 & SB2 & -473 & -305 & $\ldots$ & $\ldots$ & $\ldots$ & $\ldots$ & $2.7 \pm 0.9$ & $\ldots$ \\
\hline $\mathrm{D}(101) \ldots \ldots$ & -2.2 & -4.5 & 2D-FIS & -264 & -324 & $\ldots$ & $89 \pm 21$ & $94 \pm 6$ & $\ldots$ & $8 \pm 3$ & $0.4 \pm 0.3$ \\
\hline $\mathrm{D}(102) \ldots \ldots$ & -1.2 & -5.0 & 2D-FIS & -527 & -325 & $67 \pm 8$ & $83 \pm 2$ & $65 \pm 1$ & $46 \pm 13$ & $10.5 \pm 0.9$ & $0.12 \pm 0.03$ \\
\hline $\mathrm{D}(45) \ldots \ldots$ & -0.9 & -5.7 & SB2 & -527 & -297 & $62 \pm 12$ & $\ldots$ & $59 \pm 1$ & $\ldots$ & $10 \pm 2$ & $\ldots$ \\
\hline \multirow[t]{2}{*}{$\mathrm{D}(62) \ldots \ldots$} & -1.7 & -4.9 & SB2 & -524 & -298 & $100 \pm 35$ & $\ldots$ & $57 \pm 2$ & $\ldots$ & $7 \pm 2$ & $\ldots$ \\
\hline & & & SB2 & -256 & -298 & $\ldots$ & $\ldots$ & $60 \pm 6$ & $\ldots$ & $5 \pm 5$ & $\ldots$ \\
\hline $\mathrm{D}(63) \ldots \ldots$ & -0.6 & -4.7 & SB2 & -528 & -296 & $40 \pm 19$ & $\ldots$ & $64 \pm 2$ & $\ldots$ & $15 \pm 7$ & $\ldots$ \\
\hline $\mathrm{D}(34) \ldots \ldots$ & -7.6 & -7.7 & SB3 & -362 & -290 & $\ldots$ & $\ldots$ & $\ldots$ & $\ldots$ & $\ldots$ & $\ldots$ \\
\hline $\mathrm{D}(47) \ldots \ldots$ & -4.6 & -6.9 & $\mathrm{SB} 3^{\mathrm{e}}$ & -295 & -301 & $\ldots$ & $\ldots$ & $48 \pm 2$ & $\ldots$ & $6 \pm 2$ & $\ldots$ \\
\hline $\mathrm{D}(58) \ldots \ldots$ & -1.6 & -6.0 & SB3 & -526 & -274 & $\ldots$ & $\ldots$ & $\ldots$ & $\ldots$ & $9 \pm 5$ & $\ldots$ \\
\hline$E(10) \ldots \ldots$. & -4.3 & 5.6 & 2D-FIS & -340 & -322 & $\ldots$ & $140 \pm 47$ & $100 \pm 40$ & $86 \pm 12$ & $\ldots$ & $1.6 \pm 0.8$ \\
\hline$E(13) \ldots \ldots$. & -3.3 & 5.0 & 2D-FIS & -334 & -330 & $\ldots$ & $\ldots$ & $\ldots$ & $95 \pm 10$ & $\ldots$ & $1.8 \pm 0.5$ \\
\hline E (14) ........ & -2.3 & 5.5 & 2D-FIS & -307 & -312 & $\ldots$ & $139 \pm 23$ & $67 \pm 33$ & $\ldots$ & $0.2 \pm 0.2$ & $2.0 \pm 0.6$ \\
\hline E (19) ........ & -4.2 & 3.3 & 2D-FIS & -320 & -320 & $\ldots$ & $\ldots$ & $31 \pm 8$ & $\ldots$ & $\ldots$ & $1.0 \pm 0.7$ \\
\hline$E(20) \ldots \ldots$. & -3.3 & 3.9 & 2D-FIS & -296 & -320 & $\ldots$ & $86 \pm 24$ & $84 \pm 37$ & $86 \pm 12$ & $0.9 \pm 0.7$ & $2 \pm 1$ \\
\hline E (29) ....... & -3.2 & 2.7 & 2D-FIS & -330 & -324 & $\ldots$ & $\ldots$ & $\ldots$ & $\ldots$ & $0.7 \pm 0.6$ & $1.6 \pm 0.7$ \\
\hline$E(30) \ldots \ldots$. & -2.2 & 3.3 & 2D-FIS & -337 & -319 & $\ldots$ & $\ldots$ & $\ldots$ & $\ldots$ & .. & $1.2 \pm 0.3$ \\
\hline E (42) ........ & -2.3 & -1.2 & 2D-FIS & -336 & -318 & $\ldots$ & $51 \pm 8$ & $\ldots$ & $\ldots$ & $0.4 \pm 0.2$ & $2 \pm 1$ \\
\hline$E(164) \ldots \ldots$ & -2.7 & 2.8 & SB1 & -229 & -283 & $\ldots$ & $\ldots$ & $\ldots$ & $\ldots$ & $0.3 \pm 0.3$ & $\ldots$ \\
\hline E (127) ...... & -2.6 & 2.6 & SB2 & -321 & -303 & $\ldots$ & $\ldots$ & $\ldots$ & $\ldots$ & $\ldots$ & $\ldots$ \\
\hline E (139) ....... & -3.4 & 3.4 & SB2 & -319 & -313 & $\ldots$ & $\ldots$ & $\ldots$ & $\ldots$ & $\ldots$ & $\ldots$ \\
\hline$E(152) \ldots \ldots$ & -3.1 & 4.5 & SB2 & -329 & -287 & $54 \pm 20$ & $\ldots$ & $49 \pm 12$ & $\ldots$ & $\ldots$ & $\ldots$ \\
\hline$F(186) \ldots \ldots$ & -4.7 & 6.0 & SB2 & -329 & -287 & $\ldots$ & $\ldots$ & $67 \pm 20$ & $\ldots$ & $\ldots$ & $\ldots$ \\
\hline$F(198) \ldots \ldots$ & -3.4 & 7.4 & SB2 & -291 & -285 & $50 \pm 16$ & $\ldots$ & $42 \pm 12$ & $\ldots$ & $1.0 \pm 0.4$ & $\ldots$ \\
\hline F (199) ....... & -4.5 & 7.1 & SB2 & -301 & -301 & $58 \pm 20$ & $\ldots$ & $56 \pm 15$ & $\ldots$ & $1.6 \pm 0.8$ & $\ldots$ \\
\hline$F(208) \ldots \ldots$ & -3.2 & 8.5 & SB2 & -318 & -276 & $\ldots$ & $\ldots$ & $40 \pm 7$ & $\ldots$ & $0.6 \pm 0.3$ & $\ldots$ \\
\hline$F(94) \ldots \ldots$. & 0.2 & 11.2 & SB3 & -313 & -259 & $\ldots$ & $\ldots$ & $78 \pm 4$ & $\ldots$ & $2.4 \pm 0.4$ & $\ldots$ \\
\hline$F(95) \ldots \ldots$ & -2.8 & 10.5 & SB3 & -348 & -251 & $\ldots$ & $\ldots$ & $54 \pm 2$ & $\ldots$ & $2.1 \pm 0.3$ & $\ldots$ \\
\hline$F(107) \ldots \ldots$ & 1.0 & 14.3 & SB3 & -342 & -264 & $\ldots$ & $\ldots$ & $82 \pm 5$ & $\ldots$ & $2.6 \pm 0.4$ & $\ldots$ \\
\hline$G(28) \ldots \ldots$. & 13.6 & -1.9 & SB3 & -153 & -261 & $\ldots$ & $\ldots$ & $\ldots$ & $\ldots$ & $\ldots$ & $\ldots$ \\
\hline$G(42) \ldots \ldots$. & 10.4 & -2.7 & SB3 & -566 & -288 & $71 \pm 28$ & $\ldots$ & $\ldots$ & $\ldots$ & $16 \pm 5$ & $\ldots$ \\
\hline$G(52) \ldots \ldots$ & 7.4 & 7.7 & SB3 & -221 & -287 & $\ldots$ & $\ldots$ & $\ldots$ & $\ldots$ & $\ldots$ & $\ldots$ \\
\hline
\end{tabular}

${ }^{\text {a }}$ With respect to the $\mathrm{P} 2$ location.

b $\phi=0.9$.

${ }^{\circ} \phi=0$ ". 45 .

d $\phi=0$.'9.

e $\phi=2$ ".7.

ditions are affecting the two different kinematic subsystems of cloud D.

The spread ionized gas region toward the northwest identified in Figure 12 presents [O III] $\lambda 5007 / \mathrm{H} \beta \sim 2-4$. We have no spectra of this region in the $\mathrm{H} \alpha$ and $[\mathrm{N} \mathrm{II}] \lambda 6548$, 6584 wavelength range. But this region spatially coincides with a shell-like dust cloud 8 " in diameter located $16^{\prime \prime}$ north of the nucleus (Bacon et al. 1994). These results suggest that this is an $\mathrm{H}$ II region.

\subsection{Kinematics of the Ionized Gas Systems}

In Table 2 we present the ionized gas velocities deter- mined for each spectrum. We have compared some of these values (corresponding to the outer regions) with the velocities measured by $\mathrm{C} 88$ along different directions (P.A. $=$ $37.9,52^{\circ}, 128^{\circ}, 142^{\circ}, 173^{\circ}$ ) with a long slit and found clear correspondences (see Fig. 6 of C88). Sil'chenko, Burenko, \& Vlasyuk (1998) also reported long-slit data obtained at P.A. $=-10^{\circ}, 35^{\circ}, 55^{\circ}, 80^{\circ}, 125^{\circ}$, and $155^{\circ}$.

In Table 3 we present the mean velocities of the A, B, C, $\mathrm{D}$, and $\mathrm{E}$ ionized gas clouds (locations with respect to $\mathrm{P} 2$ are given in Table 3 ) and the stellar velocities at the location of each cloud. In the case of cloud D we considered two different velocities corresponding to each one of its two 

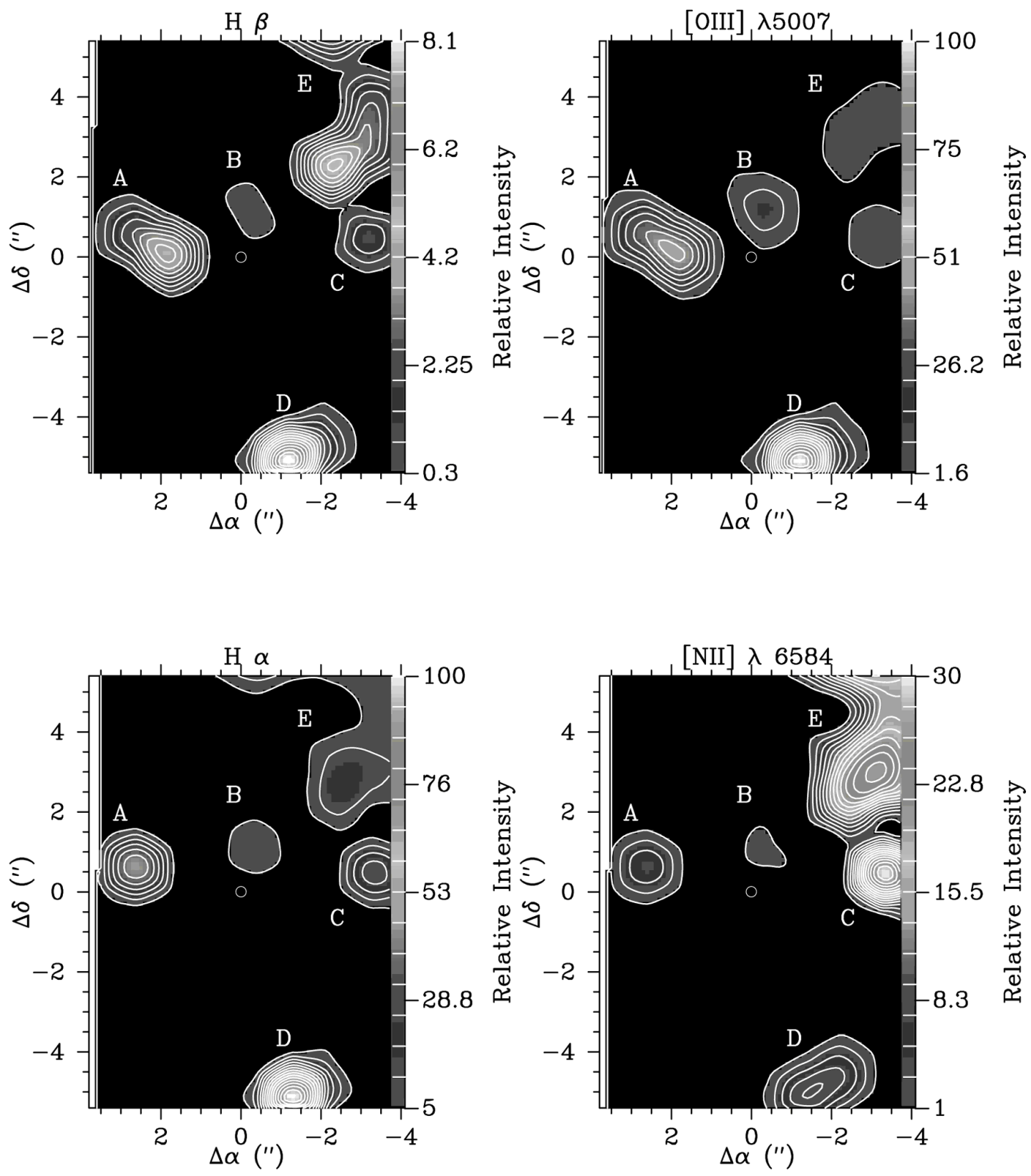

FIG. 9.-Distribution of the ionized gas clouds obtained from $\mathrm{H} \beta$ (top left), [O $\mathrm{III}] \lambda 5007$ (top right), $\mathrm{H} \alpha$ (bottom left), and [N $\mathrm{II}] \lambda 6584$ (bottom right) emission lines of M31 for 2D-FIS data.

kinematic systems. In Figure 13 we represent these values versus position angle and find that the velocity of the clouds is compatible with organized motions (especially if we adopt the most negative value or a mean value between the two velocities that correspond to cloud D). However, a strong decoupling with respect to the stellar velocities can also be noted, which implies that the ionized gas is not following the stellar rotation. In principle the ionized gas

TABLE 3

Mean Characteristic Parameters of Ionized Gas

\begin{tabular}{|c|c|c|c|c|c|c|c|c|c|}
\hline Region & $\begin{array}{c}\Delta \alpha \\
(\operatorname{arcsec})\end{array}$ & $\begin{array}{c}\Delta \delta \\
(\operatorname{arcsec})\end{array}$ & $\begin{array}{c}\text { FWHM } \\
\text { (pc) }\end{array}$ & $\begin{array}{l}\text { FWHM } \\
(\operatorname{arcsec})\end{array}$ & $\begin{array}{c}\sigma \\
\left(\mathrm{km} \mathrm{s}^{-1}\right)\end{array}$ & $\begin{array}{c}V_{\mathrm{gas}} \\
\left(\mathrm{km} \mathrm{s}^{-1}\right)\end{array}$ & $\begin{array}{c}V_{\text {stars }} \\
\left(\mathrm{km} \mathrm{s}^{-1}\right)\end{array}$ & {$[\mathrm{O} \mathrm{III}] \lambda 5007 / \mathrm{H} \beta$} & {$[\mathrm{N} \mathrm{III}] \lambda 6584 / \mathrm{H} \alpha$} \\
\hline A ...... & 2.3 & 0.3 & 2.7 & 0.7 & $56 \pm 2$ & $-56 \pm 1$ & $-318 \pm 1$ & $12 \pm 1$ & $0.14 \pm 0.10$ \\
\hline $\mathrm{C} \ldots \ldots$ & -3.0 & $0.3 \pm 0.1$ & $\sim 1.5$ & 0.4 & $58 \pm 6$ & $-464 \pm 4$ & $-310 \pm 10$ & $2.6 \pm 0.3$ & $1.0 \pm 0.2$ \\
\hline $\mathrm{D} \ldots \ldots$ & $-1.0 \pm 0.2$ & $-5.5 \pm 0.2$ & $\sim 12$ & 3.2 & $60 \pm 1$ & $-270 /-527$ & $-308 \pm 3$ & $10.5 \pm 0.9$ & $0.12 \pm 0.03$ \\
\hline $\mathrm{E} \ldots \ldots$ & $-2.2 \pm 0.2$ & $2.3 \pm 0.1$ & 2.0 & 0.5 & $58 \pm 6$ & $-318 \pm 1$ & $-306 \pm 2$ & $0.54 \pm 0.05$ & $1.5 \pm 0.1$ \\
\hline $\mathrm{F} \ldots \ldots$ & $-4.3 \pm 0.6$ & $7.7 \pm 0.6$ & $\ldots$ & $\ldots$ & $61 \pm 8$ & $-334 \pm 3$ & $-258 \pm 2$ & $1.9 \pm 0.2$ & $\ldots$ \\
\hline
\end{tabular}



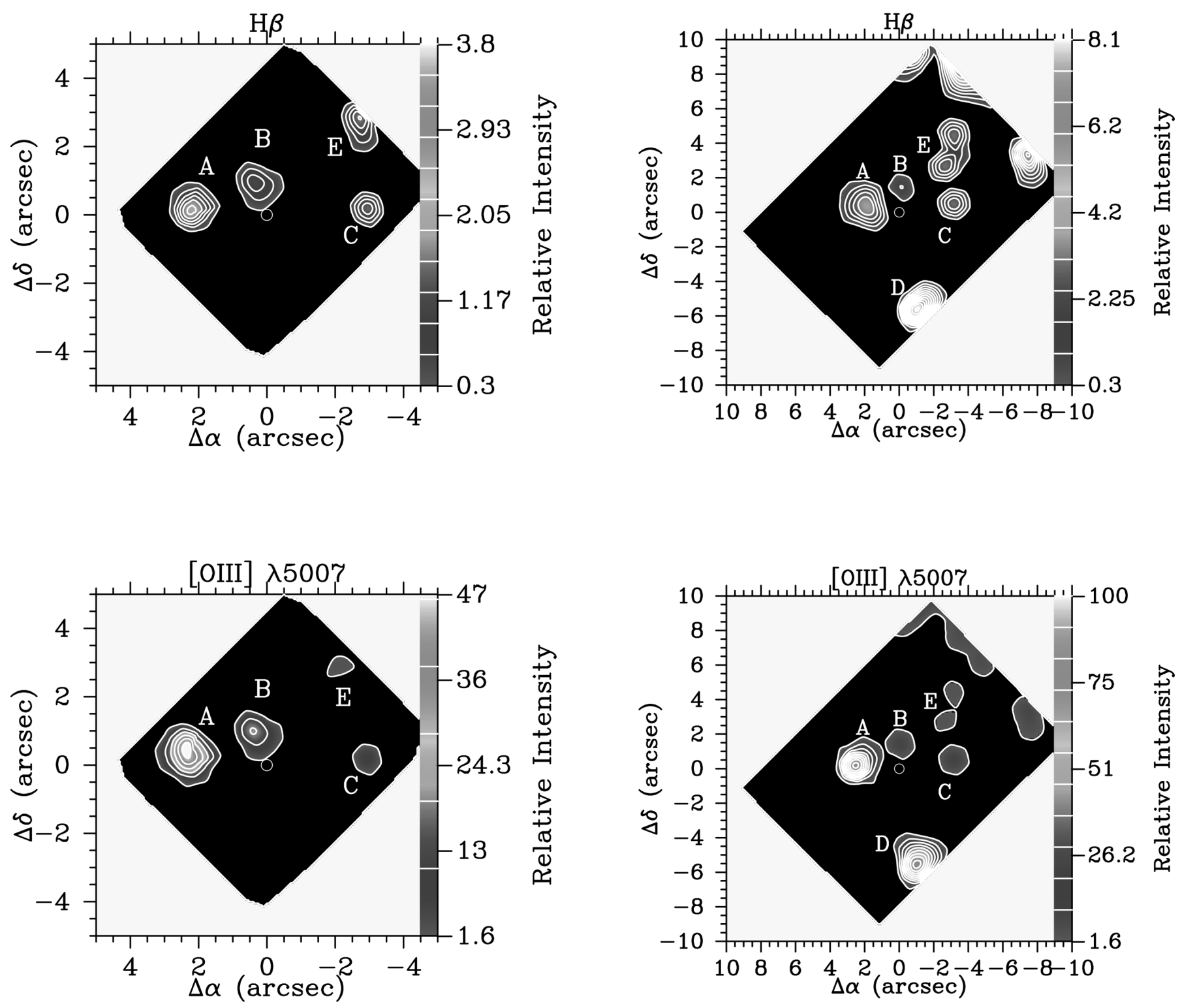

FIG. 10.-Distribution of the ionized gas clouds from $\mathrm{H} \beta$ (top) and [O III] $\lambda 5007$ (bottom) emission lines of M31 obtained using SB1.

could be rotating around a different axis (different plane) from that of the stars. Assuming a circular Keplerian velocity for the ionized gas clouds $\mathrm{A}$ and $\mathrm{C}$, we have estimated the central mass $M$ from the mean amplitude, $V=1 / 2\left(V_{A}\right.$ $\left.+V_{C}\right)$, and its mean galactocentric distance, $R=1 / 2\left(R_{A}\right.$ $+R_{C}$ ), via the expression $M=R V^{2} / G$. From this formula we obtain an inclination-dependent estimation for the mass: $M_{G} \sim 3 \times 10^{8} M_{\odot}(\sin i)^{-1}$.

We can also try to infer a mass from the stellar kinematics to compare with $M_{G}$. The relevance of rotation in the stellar kinematics changes drastically with galactocentric distance. Close to the galactic center the stars seem to be rotating very rapidly, reaching a maximum amplitude of $142 \mathrm{~km} \mathrm{~s}^{-1}$ at a galactocentric radius of 0 " 8 . At greater distances the relevance of rotation decreases progressively, being negligible at the location of clouds A and C. At this distance the stellar motion seems to be ruled by nonordered motions typical of bulge kinematics with dispersion velocities $\sigma \sim 175 \mathrm{~km} \mathrm{~s}^{-1}$. From this value we can infer, under the

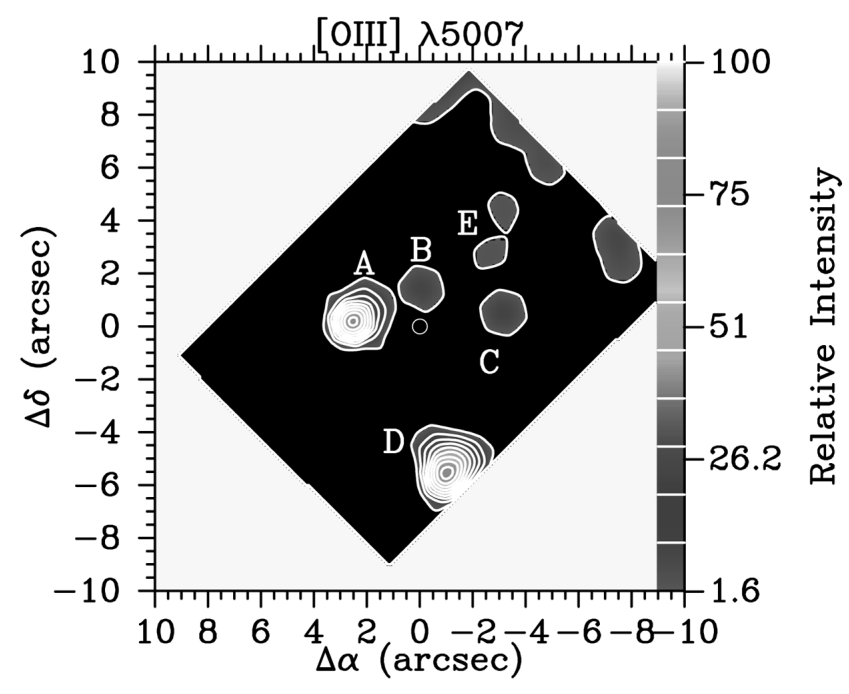

FIG. 11.-Distribution of the ionized gas clouds from $\mathrm{H} \beta$ (top) and [O III] $\lambda 5007$ (bottom) emission lines of M31 obtained using SB2.

singular isothermal sphere approximation, a mass inner to $\mathrm{A}$ and $\mathrm{C}$ of $M_{S} \sim 8.5 \times 10^{7} M_{\odot}$. Thus, even in the most favorable and limiting case, $i=90^{\circ}$, the mass inferred from the ionized gas kinematics under the hypothesis of rotation exceeds by a factor of 3.3 the mass inferred from the stellar kinematics. If, instead of this limit value for the inclination, we adopt $i \sim 77^{\circ}$ (see Sil'chenko et al. 1998), the value of $M_{G}$ could be 3.6 times greater than the value of $M_{S}$.

Then, although the central stellar kinematics of M31 is indeed complex, the comparison between $M_{G}$ and $M_{S}$ seems to indicate that the ionized gas is not rotating. Only if the decrease in the amplitude of the mean stellar velocity field with distance were caused by a strong change in inclination could the difference between $M_{G}$ and $M_{S}$ be explained.

The velocity dispersions of the different clouds are typically about $60 \mathrm{~km} \mathrm{~s}^{-1}$. This value is substantially greater than that corresponding to the ionized gas outside the circumnuclear region $\left(30-40 \mathrm{~km} \mathrm{~s}^{-1} ; \mathrm{C} 88\right)$. However, it is smaller than that of the stars $\left(\sim 140 \mathrm{~km} \mathrm{~s}^{-1}\right)$, which implies 

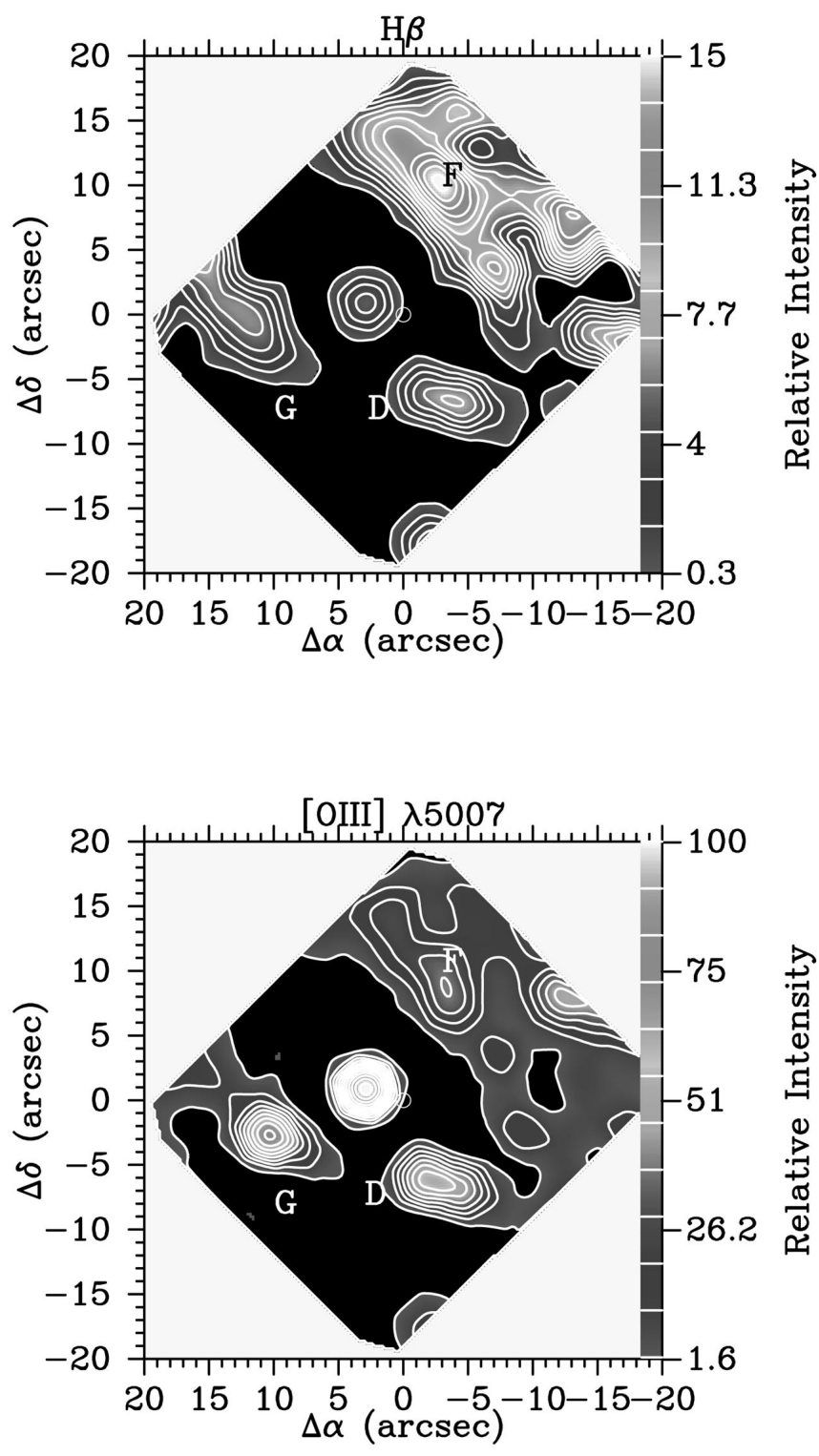

FIG. 12.-Distribution of the ionized gas clouds from $\mathrm{H} \beta$ (top) and [O III] $\lambda 5007$ (bottom) emission lines of M31 obtained using SB3.

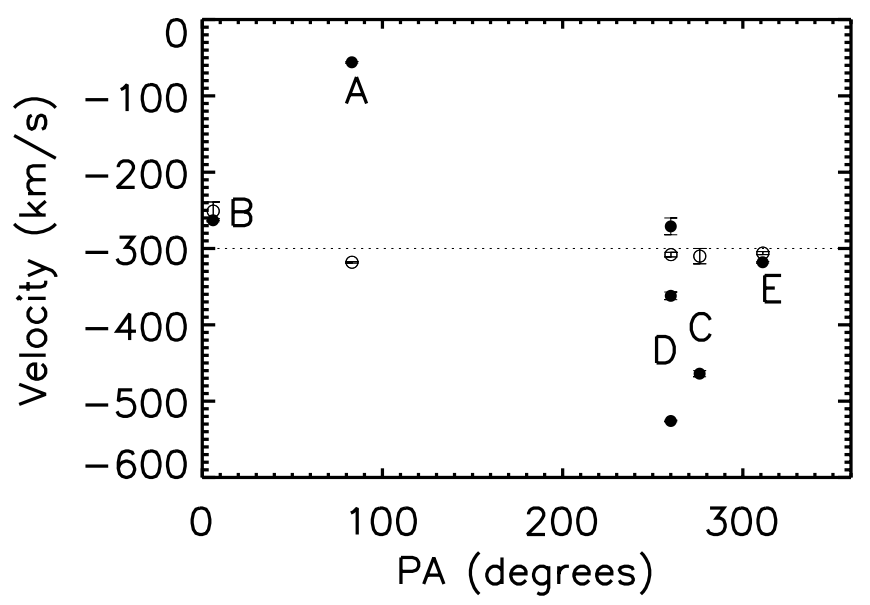

FIG. 13.- Ionized gas velocity (closed circles) and stellar velocity (open circles) versus position angle (P.A.) at the locations concerning ionized gas clouds A, B, C, D, and E. that the ionized gas is decoupled from the stellar component.

\section{DISCUSSION}

In Figure 14 we have represented the $[\mathrm{O}$ III] $\lambda 5007 / \mathrm{H} \beta$ and $[\mathrm{N} \mathrm{II}] \lambda 6584 / \mathrm{H} \alpha$ line quotients (see Table 3 ) in a diagnostic diagram with the following results: (1) clouds A, B, and $\mathrm{D}$ lie in the border of the $\mathrm{H} \mathrm{II/Seyfert} \mathrm{regions,} \mathrm{(2)} \mathrm{cloud}$ E shows typical LINER quotients, and (3) cloud C is sited at the low-ionization end of the Seyfert galaxies. In the same diagram we have included the quotients corresponding to the diffuse ionized gas (DIG) in M31 (Greenawalt, Walterbos, \& Braun 1997). As we can see, different ionization mechanisms seem to be present in M31. The possible LINER characteristics of the ionized gas in the central kiloparsec of M31 have been discussed by H96. The present results confirm this supposition.

The highest ionization emission corresponding to clouds $\mathrm{A}, \mathrm{B}$, and D, however, has passed unnoticed by previous authors. This is probably due to its absence in the nucleus and to its presence in only three isolated clouds, which greatly complicates the detection by means of long-slit spectroscopy. In addition, the detection of these clouds requires the subtraction of a high signal-to-noise representative absorption spectrum. This goal is more easily achieved with the aid of two-dimensional spectroscopy. We have considered a possible offset of the emission-line quotients due to a high extinction along the line of sight at the ionized gas cloud positions. In order to get an offset of 0.4 dex along the [O III] $\lambda 5007 / \mathrm{H} \beta$ axis, an extremely large extinction in the visible is required, $A_{V}=25$, assuming the $A_{\lambda} / A_{V}$ ratios of Mathis (1990). Thus, we can hardly explain the emissionline quotient locations as a consequence of reddening by dust.

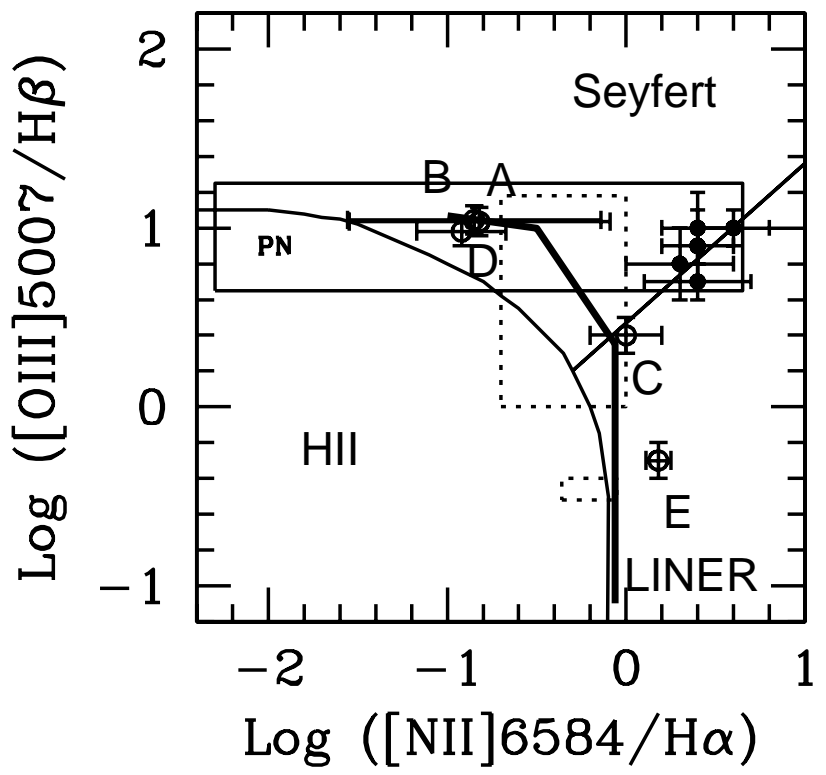

FIG. 14. - Diagnostic diagram $\log [\mathrm{O}$ III $] \lambda 5007 / \mathrm{H} \beta$ versus $\log [\mathrm{N}$ II $]$ $\lambda 6584 / \mathrm{H} \alpha$ with the $\mathrm{A}, \mathrm{B}, \mathrm{C}, \mathrm{D}$, and $\mathrm{E}$ ionized gas clouds of M31 (open circles), NGC 4151 (big dashed-line rectangle), and DIG of M31 (small dashed-line rectangle). Also marked are the Seyfert, LINER, H II, and PN regions. The limits of the Seyfert galaxies, LINERs, and $\mathrm{H}$ II regions are the same as those of Véron, Gonçalves, \& Véron-Cetty (1997). A power-law model (thick line) with $\alpha=-1.5$ (Hill et al. 1999) is also represented (see text for details). 
It has been suggested that post-asymptotic giant branch (PAGB) stars could be a source of photoionization acting in M31 (H96). In fact, King et al. (1992) identify a sprinkling of PAGB stars contributing almost a fifth of the total UV light in the central $44^{\prime \prime}$ of M31. Moreover, planetary nebulae (PNs) share a wide common region with Seyfert galaxies in the diagnostic diagram (see Fig. 14), and clouds A, B, and D lie well within the PN region. Some bright UV sources are easily identified from the HST Faint Object Camera (FOC) images obtained in different wavelength ranges (F175W and F275W). Comparing our maps with Figure 7 of Lauer et al. (1998), we find that some of these objects are inside the borders of our clouds.

To study the PAGB stars as possible ionization sources, we have estimated the $\lambda 5007$ fluxes $\left(F_{\lambda 5007}\right)$ of the clouds A, $\mathrm{B}$, and C from the SB1 and 2D-FIS data. To calibrate our system the surface brightness profile of M31 (Lauer et al. 1993) and a value of $A_{V}=0.24$ (Burstein \& Heiles 1984) have been taken into account. For cloud A, we obtained $F_{\lambda 5007}=1.5 \pm 0.5 \times 10^{-12} \mathrm{ergs} \mathrm{s}^{-1} \mathrm{~cm}^{-2}$. For cloud B, $F_{\lambda 5007}=2.75 \pm 0.75 \times 10^{-13} \mathrm{ergs} \mathrm{s}^{-1} \mathrm{~cm}^{-2}$. Finally, for cloud $\mathrm{C}, F_{\lambda 5007}=5 \pm 1 \times 10^{-14} \mathrm{ergs} \mathrm{s}^{-1} \mathrm{~cm}^{-2}$. These values should be considered as lower limits because they are corrected neither for light losses in the interfiber regions nor for extinction. It is important to note that the planetary nebula in the bulge of M31 ranges from $F_{\lambda 5007}=2.4$ $\times 10^{-14}$ to $4.92 \times 10^{-16} \mathrm{ergs} \mathrm{s}^{-1} \mathrm{~cm}^{-2}$ (Ciardullo et al. 1989). Hence, clouds A, B, and C are $\sim 100,10$, and 2 times more luminous than the most luminous planetary nebula. This result seems to exclude the PAGB stars as the highionization source for cloud A, although clouds B and C need a more accurate study (perhaps considering the combined effect of several PNs).

According to the line quotients of clouds A, B, and C, M31 may be classified as a Seyfert 2 galaxy. It would seem to be a rather atypical Seyfert galaxy in which weak emission lines of relatively small broadening are situated on top of a continuum of stellar origin; however, these characteristics can also be found in the extended narrow-line region (ENLR) of the Seyfert galaxies. For instance, in prototype Seyfert galaxy NGC 4151, the spectra of the ENLRs are characterized by an undiluted stellar continuum (as in the case of M31) and present very narrow (FWHM $\sim 30 \mathrm{~km}$ $\mathrm{s}^{-1}$ ) and weak emission lines (Schulz, Fried, \& Hippelein 1983; see also Fig. 3 of Robinson et al. 1994). (In Fig. 10 we have also represented the line quotients corresponding to the ENLR of NGC 4151.) This region is currently interpreted as disk-gas-photoionized by the continuum of the active nucleus. M31 could be a scaled-down version of this scenario, where an ultralow luminous nuclear source illuminates a comparatively tiny region of influence. There are several lines of kinematic evidence supporting the hypothesis that a compact nucleus (possibly a supermassive black hole of $\sim 3 \times 10^{7} M_{\odot}$ ) is located at the center of mass of M31 (Bacon et al. 1994; Kormendy \& Richstone 1995). King et al. (1995) proposed the existence of a compact nonthermal UV source at this location. However, it seems that this source is spatially resolved (Lauer et al. 1998; Brown et al. 1998) and cannot be assumed to be a compact AGN.

Leaving aside the discussion about the optical counterpart for the AGN, it is interesting to note that a power-law model taken from Hill et al. (1999) with exponent $\alpha=-1.5$, a depleted solar metallicity, and an electron density of $10^{3}$ $\mathrm{cm}^{-3}$ fits the five clouds quite well, with only the ionization parameter varying (see Fig. 14). The differences in the emission-line quotients among the different clouds could be explained as the result of their different locations with respect to the central ionizing engine.

The detection of high-ionization nuclear activity in M31 opens up the possibility of observing a similar phenomenon in other galaxies of low activity in which the presence of a supermassive black hole is also suspected. In principle, from the statistics and past evolution of quasars, a large population of galactic nuclei including a "dead quasar" is expected, and, in fact, LINERs have been supposed to be a signature of this kind of object (e.g., H96). However, there are several factors, like the absence of gas that can be ionized, or masking by other ionization sources (notice that among the different ionization mechanisms acting in the circumnuclear region of M31, the Seyfert one seems to be the faintest), that could make difficult the detection of gas ionized by the active nucleus. In any event, two-dimensional spectroscopy in the circumnuclear regions of lowluminosity active nuclei offers an interesting way of studying the "dead quasar problem."

\section{CONCLUSIONS}

We have obtained two-dimensional spectroscopy of the central region of M31, which has allowed us to study the stellar and ionized gas components. We note the following results and conclusions:

1. We have discovered line emission in the inner $\left(r \leq 5^{\prime \prime}\right)$ circumnuclear region. The ionized gas producing these lines is distributed in several clouds. Attending to their emissionline quotients, several clouds exhibit high-ionization activity. In others we found LINER or intermediate LINER/Seyfert characteristics. The existence of circumnuclear activity is an additional piece of evidence supporting the existence (proposed on other grounds) of a supermassive black hole, a dead quasar, in the nucleus of M31.

2. The kinematics of the clouds seems to be organized, but there is a strong decoupling with respect to the stellar component. Comparison with the star's velocity field indicates that the ionized gas kinematics cannot be straightforwardly interpreted in terms of rotation.

3. The coexistence of different ionization mechanisms (Seyfert-like, LINER, H II) in the circumnuclear region of M31 underlines the hybrid character of the LINER phenomenon.

We would like to thank Reynier Peletier and the anonymous referee for their suggestions. Thanks are also due to Luis Cuesta for the code GRAFICOS and to Terry Mahoney for his help editing the manuscript. We also thank all the staff at the ING/ORM and the INTEGRAL team at the IAC for their kind support. This work has been partially supported by the DGYCIT (PB98-0340). 


\section{REFERENCES}

Arribas, S., et al. 1998, Proc. SPIE, 3355, 821

Bacon, R., Emsellem, E., Monnet, G., \& Nieto, J. L. 1994, A\&A, 281, 691

Brown, T. M., Ferguson, H. C., Stanford, S. A., \& Deharveng, J.-M. 1998, ApJ, 504, 113

Burstein, D., \& Heiles, C. 1984, ApJS, 54, 33

Carter, D., et al. 1993, ISIS User's Manual (vers. 1.0; La Palma: Isaac Newton Group)

Ciardullo, R., Jacoby, G. H., Ford, H. C., \& Neill, J. D. 1989, ApJ, 53, 69

Ciardullo, R., Rubin, V. C., Jacoby, G. H., Ford, H. C., \& Ford, W. K., Jr. 1988, AJ, 95, 438 (C88)

Dressler, A., \& Richstone, D. O. 1988, ApJ, 324, 701

García, A., Rasilla, J. L., Arribas, S., \& Mediavilla, E. 1994, Proc. SPIE, 2198,75

Garcia, M. R., et al. 2000, ApJ, 537, L23

Greenawalt, B., Walterbos, R. A. M., \& Braun, R. 1997, ApJ, 483, 666

Heckman, T. M. 1996, in ASP Conf. Ser. 103, The Physics of LINERs in

View of Recent Observations, ed. M. Eracleous et al. (San Francisco: ASP), 241 (H96)

Hill, T. L., Heisler, C. A., Sutherland, R., \& Hunstead, R. W. 1999, ApJ, 117,125
King, I. R., et al. 1992, ApJ, 397, L35

King, I. R., Stanford, S. A., \& Crane, P. 1995, AJ, 109, 164

Kormendy, J. 1988, ApJ, 325, 128

Kormendy, J., \& Richstone, D. O. 1995, ARA\&A, 33, 581

Lallemand, A., Duschene, M. \& Walker, M. F. 1960, PASP, 72, 76

Lauer, T. R., et al. 1993, AJ, 106, 1436

Lauer, T. R., Faber, S. M., Ajhar, E. A., Grillmair, C. J., \& Scowen, P. A. 1998, AJ, 116, 2263

Light, E. S., Danielson, R. E., \& Schwarzschild, M. 1974, ApJ, 194, 257

Mathis, J. S. 1990, ARA\&A, 28, 37

Mediavilla, E., Arribas, S., García-Lorenzo, B., \& del Burgo, C. 1997, ApJ, 488,682

Robinson, A., et al. 1994, A\&A, 291, 351

Rubin, V. C., \& Ford, W. K., Jr. 1971, ApJ, 170, 25

Schulz, H., Fried, J., \& Hippelein, H. 1983, IAU Circ., 3813, 2

Sil'chenko, O. K., Burenkov, A. N., \& Vlasyuk, V. V. 1998, A\&A, 337, 349

Stanek, K. Z., \& Garnavich, P. M. 1998, ApJ, 503, L131

Statler, T. S., King, I. R., Crane, P., \& Jedrzejewski, R. I. 1999, ApJ, 117, 894

Véron, P., Gonçalves, A. C., \& Véron-Cetty, M.-P. 1997, A\&A, 319, 52

Note added in proof.- - In the lapse of time transcurred within the reception and final acceptance of this paper the X-ray Chandra Observatory has detected X-ray emission coming from a source coincident with the nuclear radio source of M31 (Garcia et al. 2000). Although the nature of this nuclear source has to be determined, its existence is in consonance with the AGN hypothesis suggested by us. 